\title{
LA ACTIVIDAD INFANTIL EN LA PRODUCCIÓN DEL REGISTRO ARQUEOLÓGICO DE CAZADORES-RECOLECTORES
}

Gustavo G. Politis*

\section{Introducción}

Los estudios arqueológicos consideran en general a las poblaciones en su conjunto y sólo en sociedades complejas se ha prestado atención a las diferencias de clase y/o status. En general, los arquéologos se han sentido poco atraídos por estudiar a los "individuos" en particular por considerarlos metodológicamente inaccesibles (Shennan 1991). Sin embargo, entre la población y el individuo, hay facciones y segmentos sociales que pueden ser reconocibles en el registro arqueológico: conjuntos de especialistas (i.e. ceramistas, orfebres, etc.), elites, hombres, mujeres, etc. El abordaje desde la arquelogía de esta heterogenidad en la conformación de la sociedad ha tenido un auge significativo a partir de los estudios de género. Recientemente también se ha dirigido la atención sobre los grupos de edad, especialmente sobre los niños, y se ha alertado sobre su contribución al registro arqueológico (Lillehammer 1989, Sofaer Derevenski 1994). Básicamente se ha reconocido explicitamente algo absolutamente obvio: que los niños son tanto productores como consumidores de cultura material.

A pesar de este tardío reconocimiento de los derivados de la actividad infantil, en la mayoría de los casos en la interpretación tanto de los sitios de cazadores-recolectores, como de otro tipo de sociedades más complejas, todavía se asume que los restos materiales recuperados (fundamentalmente los elementos tecnológicos y los desechos derivados de su confección y uso) han sido producidos por adultos. Los agentes son adultos por ausencia (default) de otros actores sociales. A partir de

(*) CONICET y Universidades del Centro de la Pcia. de Buenos Aires y de La Plata, Argentina. esta asunción implícita se han realizado inferencias sobre las conductas del pasado y se han propuesto modelos de diverso orden referentes a organización tecnológica, secuencia de producción y uso, maximización de materia prima, áreas de descarte, etc. Dentro de este marco, la forma, la tecnología de confección y el tamaño de algunos artefactos (por ej. puntas de proyectil) son considerados como una expresión idiosincrásica y utilizados para la reconstrucción histórico-cultural o como una medida de variabilidad y distancia cultural. En estas reconstrucciones, basadas en supuestos agentes adultos, los niños no han sido visualizados como actores sociales y su producción material no se ha tenido en cuenta en el estudio arqueológico de los grupos cazadores-recolectores. En la mayoría de los casos, los niños han sido habitualmente identificados en contextos mortuorios y la edad fue tratada como una variable y no como un principio fundamental de la organización social (Sofaer-Derevenski 1994). Hay algunas excepciones en la arqueología mundial que han propuesto que determinados objetos, o conjuntos artefactuales, especialmente líticos, fueron confeccionados o usados por niños (por ejemplo Frison 1970, Bodú el al. 1990, Fisher 1990, Dawe 1997, Park 1998). En América del Sur, la asignación infantil de algunos conjuntos y objetos es aún más rara. En un rápido análisis de la bibliografía existente se han encontrado muy pocas excepciones. Por ejemplo, Gradín y Aguerre (1983) identificaron negativos negros y blancos de manos en el área del Río Pinturas en la Patagonia Argentina y los asignaron a niños. En la costa central de Chile, se han hallado figurillas de greda en miniatura, en sitios de la tradición Aconcagua, que fueron interpretadas como juguetes de niños (Rivas y Ocampo 1997). En varios sitios de la Región Pampeana de Argentina se ha planteado que algunas puntas de proyectil, bolas de boleadores y rodados basálticos 
podrían haber sido producidos o usado por los niños de las sociedades cazadoras-recolectoras prehispánicas que habitaron la región (Politis 1998). Por último, en las Terceras Jornadas de Arqueología de la Patagonia, se llevó a cabo una mesa redonda, titulada Actores en escena: comportamiento social y registro arqueológico, en donde se dió una discusíon acerca de la agencia infantil en la generación de sitios arqueológicos (Mengoni Goñalons en prensa). Seguramente hay más ejemplo, pero estos son algunos de los pocos que ilustran las escasas referencias a objetos asignados a niños.

A pesar de estos estudios puntuales, no ha habido programas de investigación sistemáticos destinados a contribuir al conocimiento del agente infantil en las sociedades del pasado (para excepciones ver Dawe 1997, Park 1998, Politis 1998). Tampoco se ha tratado de identificar la actividad de los niños en la creación de los espacios domésticos y en la formación de sitios. Algunos autores han alertado sobre la acción de los niños en los depósitos, pero desde una perspectiva estrecha ya que los han visto como agentes distorcionadores y perturbadores de los materiales depositados por los adultos (Bonnichsen 1973, Hammond y Hammond 1981). En estos trabajos los niños han sido considerados como cualquier otro agente biológico post-depositacional pre-enterramiento, o sea modificando un registro arqueológico "normal" producido por los adultos. Este enfoque es erróneo por que asume que los niños no generan registro arqueológico, sino que sólo lo perturban. No son considerados como productores y consumidores de cultura material, ni tampoco como actores sociales.

Como consecuencia de los aportes de la arqueología post-procesual, cada vez con mayor intensidad se esta tratando de generar una visión de pasado que sea socialmente mas inclusiva y que permita recurperar la variabilidad interna del registro resultante de la participación de diferentes - géneros y grupos de edad. El naciente interés arqueológico por detectar la actividad infantil es una derivación de la consideración de los niños como actores sociales significativos (James y Prout 1990). En concordancia con esto, algunos trabajos recientes han enfatizado que : "... that children contribute to the archaeological record, whether or not we are competent in recognize them (Chamberlain 1997:249, énfasis en el original).

En trabajos anteriores plantié que los niños (de aqui en adelante, salvo cuando se exprese lo contra- rio, "niños" será usado en sentido amplio, incluyendo tanto varones como mujeres) son generadores importantes de residuos materiales dentro de los campamentos de cazadores-recolectores y que su actividad ha sido subestimada en el análisis e interpretación de los depósitos arqueológicos (Politis 1998; Politis y Saunders en prensa). Además, en el primero de estos artículos comparé las expectativas arqueológicas generadas a partir del estudio de los Nukak con el registro de los sitios arqueológicos de la Región Pampeana.

De alguna manera, este intento de considerar a los niños como actores sociales y de dotarlos de visibilidad arqueológica, se asemeja a los inicios de la arqueología del género cuando hace más de quince años propuso identificar el protagonismo de la mujer en las sociedades del pasado y llamar la antención sobre el sesgo androcéntrico de la interpretación arqueológica (Conkey y Spector 1984, Gero 1985). Sin embargo hay una diferencia que quiero señalar. La arqueología del género (básicamente del femenino) nos ha enseñado que las mujeres deben ser estudiadas como participantes activos dentro de la sociedad, como agentes y sujetos (Conkey y Gero 1991: 6) y no sólo en relación a los hombres o dentro del contexto masculino. También ha hecho notar el sesgo genérico en las reconstrucciones que la arqueología ha hecho del pasado (el gender bias de la literatura anglo-sajona). Sin embargo y a pesar de la cada vez más copiosa literatura referente a los estudios de género en arqueología, aún no se ha desarrollado un cuerpo metodológico significativo que permita reconocer adecuadamente lo femenino o lo genérico dentro del registro arqueológico (para excepciones ver por ejemplo Gero 1991). Esto ha llevado a plantear con cierta desilución que: "A closer look, however, reveals that the 'gender revolution' in archaeology is only just beginning now" (Lesik 1997) o ya en forma más contundente que: "In spite of 20 years of the new archaeology, a decade of postprocessualism and furious 5 years of feminst archaeology/ anthropology of gender, we still approach artifacts, ecofacts, features and their matrix in essentially the same way we have for 100 years"(Claassen 1992:6).

En el caso de la arqueología de la infancia, lo que pretendo hacer en éste trabajo, no es sólo llamar la atención sobre la actividad de los niños en las sociedades del pasado, sino entregar algunas herramientas metodológicas para poder identificarla adecuadamente en el registro material. Este 
será el primer paso para analizar y discutir luego la agencia infantil en estas sociedades y para explorar los mecanísmos escenciales de la transmición cultural desde una perspectiva temporal amplia. Desde ya, voy a dejar de lado las consideraciones relacionadas con el entrenamiento para la vida adulta que tienen todos los juegos, ya que esto es un hecho obvio, ni tampoco trataré la participación de los niños en la subsistencia del grupo. Ambos temas, si bien son interesantes y estan interrelacionados, exeden los objetivos de este artículo.

En investigaciones recientes se ha remarcado que el género es un concepto relacionado con la edad. El sexo esta biológicamente determinado, pero el género es una construcción social. De esta manera, se ha observado que: "Ignoring the temporal of gender has led archaeologists to impose a static dualistic vision of gender onto the past through straightforward artefact association" (SofaerDerevenski 1997:877). El reconcimiento de esta dimensión temporal en los estudios de género ha cambiado totalmente el enfoque, ya que se lo dejó de ver como una categoría o unidad de análisis y se lo comenzó a considerar como un proceso que se extiende durante toda la vida (Lorber 1994 en Sofaer-Derevenski 1997). Actualmente se considera que el aprendizaje del género es continuo y esta estrechamente relacionado con la edad.

La arqueología de la infancia es novedosa y tiene una historia muy reciente. Algunos conceptos básicos relacionados a los niños ya fueron discutidos previamente por Lillehammer (1989) en base a una revisión de la arqueología escandinava, en un artículo pionero dentro del tema. Dentro de esta misma línea de análisis una serie de trabajos han arribado a conclusiones similares desde perspectivas diferentes. Entre estos aportes se destaca el libro recientemente editado por Moore y Scott (1997) sobre aspectos teóricos y metodológicos y las contribuciones de Dawe (1997) y Park (1998) con originales análisis de casos de estudio arqueológicos de las llanuras norteamericanas y de los Inuit de Canadá. El resultado de estos estudios indica que los productos de la actividad infantil pueden ser reconocidos si se desarrolla una metodología apropiada. Para la construcción de esta metodología la argumentación analógica juega un papel central y las principales fuentes deben buscarse en los grupos humanos actuales, y en la información histórica y etnográfica. Este trabajo apunta a contribuir al desarrollo de instrumentos metodológicos para identificar e interpretar el agente infantil en las sociedades del pasado y se basa en una argumentación analógica usando como fuente a los $\mathrm{Nu}$ kak y, de manera complementaria a otros grupos cazadores-recolectores de América del Sur: Guayakí de Paraguay, Sirionó de Bolivia, Tehuelches de la Patagonia Argentina, Selknam de la Tierra del Fuego y Yámanas de los Canales Fueginos del extremo sur Americano (Figura 1). La información obtenida entre los Nukak (un grupo cazadorrecolector-pescador de la Amazonia colombiana), entre 1990 y 1996 ( 7 temporadas de campo, 185 días en total) permite generar expectativas materiales de la actividad infantil y evaluar bajo que condiciones se puede esperar, en sociedades análogas del pasado, un contexto similar.

Para los fines de este trabajo, cuando me refiero a los niños Nukak estoy incluyendo a individuos cuyo rango de edad varía desde el tiempo que empiezan a caminar, hasta la pubertad. En términos de años esto correspondería al período entre a uno a dos años y 12 a 13 años. Con anterioridad a este lapso los bebés dependen enteramente de sus padres y no generan ningún tipo de artefacto (aunque si hay algunos que son confeccionados para ellos como por ejemplo los collares de diente de mono); en la mayoría de los casos aún no tienen nombres y se los llama genéricamente jim 'bú o tóm 'bú según el sexo. A partir de la pubertad, los jovenes Nukak salen de cacería y realizan todas las actividades de los adultos aunque aún no hayan completado el proceso de aprendizaje. En términos económicos, su rol es similar al de los adultos, a pesar de no pertenecer aún a ese grupo de edad y aunque socialmente todavía tengan diferencias con éstos. A los 15 o 16 años un joven Nukak es económicamente tan productivo como un adulto. En el caso de las púberes ocurre lo mismo, aunque como forman pareja más temprano, el rol social que desempeñan se transforma más rapidamente, aproximándose más al de mujeres adultas (estan casadas, pueden quedar embarazadas, realizan todas las tareas femeninas en el campamento, etc.).

El rango de edad que abarca la infancia parece ser variable entre las sociedades indígenas. En algunos casos es muy semejante a los Nukak, como por ejemplo entre los Guayaquí (Clastres 1972), Yámanas (Gusinde 1983), Sirionó (Holmberg 1978) y Ayoréo (Bórmida 1973). Para estos últimos la palabra disiehóde significa "niños" y abarca desde el nacimiento hasta el comienzo de la pubertad, 
POLITIS, G.G. La actividad infantil en la producción del registro arqueológico de cazadores-recolectores. Rev. do Museu de Arqueologia e Etnologia, São Paulo, Suplemento 3: 263-283, 1999.

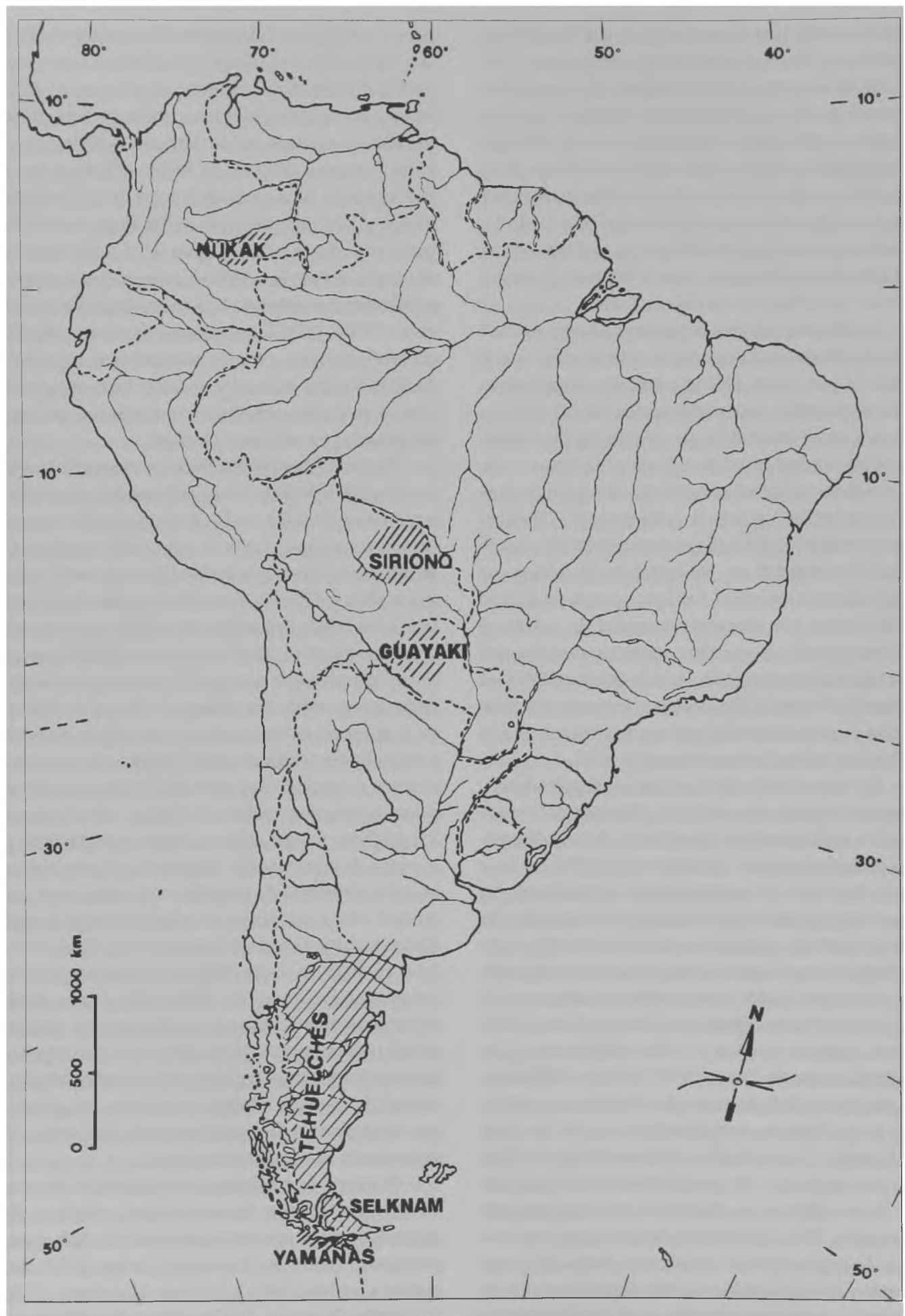

Fig. 1-Mapa de América del Sur con la ubicación de los grupos cazadores-recolectores mencionados en el texto. 
cerca de los 12 años (Bórmida 1973: 64). Por el contrario, entre los Hadza orientales, Hawkes el al. (1995: 689) incluyen bajo la denominación "children" a varones de hasta 17 años, una edad en la cual un joven Nukak ya es plenamente productivo y utiliza artefactos semejantes a los de los adultos.

\section{Algo acerca de los Nukak}

Los Nukak son indígenas de filiación Makú (Metraux 1948, Silverwood-Cope 1972, Reid 1979) que habitan la Amazonia colombiana, en el interfluvio de los ríos Guaviare e Inirida, en el Depto. del Guaviare (Figura 1). En un territorio de aproximadamente $10.000 \mathrm{~km}^{2}$, viven entre 400 y $500 \mathrm{Nu}$ kak que se agrupan en bandas de unos 20 a 30 individuos (en el sector nororiental hay agrupaciones mayores que pueden llegar a 50 personas). Esta región es una zona de divisoria entre las cuencas del Orinoco y el Amazonas con cobertura vegetal típica de la foresta tropical lluviosa, aunque se observa un período seco entre diciembre y marzo. Las precipitaciones fluctúan entre 2500 y $3000 \mathrm{~mm}$ anuales.

Si bien hasta 1988 había algún conocimiento sobre la existencia de indígenas de filiación Makú en el interfluvio Guaviare-Inirida (ver Reichel-Dolmatoff 1967 e Informes Inéditos de la Asociación Nuevas Tribus de Colombia), es recién en ese año cuando los antropólogos y el público en general tuvieron las primeras noticias de la existencia de los Nukak (Chaves y Wirpsa 1988). A partir de fines de la década del ' 80 , los Nukak comenzaron contactos regulares con los colonos (campesinos de otras regiones de Colombia), llegando incluso hasta la capital departamental, San José del Guaviare. Desde ese momento se aceleró el proceso de aculturación y transformación de los patrones tradicionales de los Nukak (Ardila y Politis 1992, Cabrera et al. 1994: 426-436, Politis 1996b: 355-378).

A partir de los primeros contactos, se iniciaron diferentes investigaciones antropológicas que han esbozado las características generales de la etnía. Entre los estudios más importantes merecen destacarse las investigaciones sobre el territorio (Mondragón ms., Cárdenas y Politis en prensa), organización social y parentesco (Cabrera et al. 1994, Franky et al. 1995), subsistencia (Politis y Rodríguez 1994, Cabrera et al. 1994, Politis 1996b, Politis et al. 1997, Mondragón ms.), movilidad y asentamiento (Politis 1992, 1996a y b), lingüística (Reina 1990,
Cabrera et al. 1994, Mondragón ms.) y ideología y cosmovisión (Cabrera et al. 1994, Politis 1996b, Politis y Saunders en prensa). Asimismo se debe mencionar la copiosa información inédita recogida por los misioneros de la Asociación Nuevas Tribus de Colombia, quienes hicieron los primeros contactos con algunas bandas Nukak a fines de los ' 70 .

El ciclo anual de subsistencia ha podido ser reconstruido en base a los trabajos citados y se ha estimado que la economía de los Nukak se basa en la caza (monos, pecaríes, aves etc.), la recolección de especies silvestres y de otros productos animales como la miel, los huevos de tortuga y el mojojoy (larvas de coleópteros del género Rhynchophorus). Algunos animales son tabú (jaguar, venado, tapir, etc.) y su caza y consumo esta estrictamente prohibida para todos los miembros de la banda (Politis y Saunders en prensa). Hasta el presente se han identificado 123 especies vegetales utilizadas por los Nukak (Cárdenas y Politis en prensa). La pesca y la horticultura en pequeña escala complementan las actividades económicas, con variada importancia según las estaciones. En forma creciente, en los últimos años, los Nukak están incorporando también alimentos que obtienen de los colonos. Sin embargo, durante el período de estudio la subsistencia tradicional de los Nukak se basó esencialmente en la explotación de recursos vegetales y animales no domesticados (Politis 1996b: 58).

Los campamentos residenciales son los más frecuentes dentro del sistema de asentamiento. Durante el invierno, estos campamentos estan cubiertos con hojas de platanillo y tienen una planta aproximadamente geométrica regular (triangular, cuadrangular y pentagonal). Estos asentamientos son pequeños y compactos $\left(\mathrm{n}=12, \min =32.5 \mathrm{~m}^{2}\right.$, $\max =114 \mathrm{~m}^{2}, x=3,89 \mathrm{~m}^{2} /$ persona). En el verano, la planta es irregular y no existe la cobertura de hojas. En esta estación los campamentos tienen dimensiones levemente superiores a los del invierno $\left(\mathrm{n}=8, \min =45 \mathrm{~m}^{2}, \max =129,9 \mathrm{~m}^{2}, x=4,46 \mathrm{~m}^{2} /\right.$ persona). En los casos en que hay más de una banda residiendo juntas, los asentamientos se hacen más compactos y más densamente poblados $(\mathrm{n}=4$, $\min =99 \mathrm{~m}^{2}, \max =130 \mathrm{~m}^{2}, x=2,61 \mathrm{~m}^{2} /$ persona $)$.

La movilidad residencial de los Nukak es muy alta y se ha estimado que la mudanza del campamento residencial se produce entre 70 y 80 veces al año. Durante nuestros trabajos de campo hemos registrado distancias entre campamentos residenciales (el abandonado y el nuevo) que varían entre 
POLITIS, G.G. La actividad infantil en la producción del registro arqueológico de cazadores-recolectores. Rev. do Museu de Arqueologia e Etnologia, São Paulo, Suplemento 3: 263-283, 1999.

0,9 y $18,1 \mathrm{~km}(\mathrm{n}=25, x=6,25 \mathrm{~km}$, ver Tabla 3.1 en Politis 1996b). Hay un patron diferencial entre la movilidad residencial de invierno (estación lluviosa) y la de verano (estación seca). En la primera, la distancia entre campamento es de $X=3,85 \mathrm{~km}(\mathrm{n}=12)$ y el promedio de ocupación es de 5 dias $(n=13)$, mientras que en la segunda la distancia es $X=$ $8,94 \mathrm{~km}(\mathrm{n}=13)$ y el promedio de ocupación es de 3 dias $(n=15)$.

Por otro lado, la movilidad logística diaria (considerada mas allá del área que rodea el campamento - aproximadamente un radio de $1 \mathrm{~km}$ ) mantiene un patron similar en ambas estaciones. En invierno $X=8,52 \mathrm{~km}(\mathrm{n}=14, \min =3,50 \mathrm{~km}, \max =$ $19,19 \mathrm{~km})$ y en verano $X=8,30 \mathrm{~km}(\mathrm{n}=13, \min =$ $3 \mathrm{~km}, \max =14,56 \mathrm{~km})$. Esto dá para ambas estaciones un $X=8,41 \mathrm{~km}(\mathrm{n}=27 \mathrm{~km})$.

Los Nukak estan organizados en bandas autónomas, vinculadas a grupos mayores de afiliación que tienen nombres definidos en referencia a su ubicación dentro del territorio (i.e. Wayari los del nororiente, Tákayu los del centro, Muhabeh los del suroriente, $\mathrm{Meu}$ los del noroccidente etc.). Cada banda esta formada por pocas familias, usualmente no más de 5 y excepcionalmente hasta 8 . El tama- ño máximo de una banda, registrado en nuestros trabajos de campo fue de 46 individuos y el mínimo 12 (aunque este último valor esta representando probablemente un segmento de una banda). En ocasiones especiales dos bandas (o segmentos) pueden acampar juntas por pocos días, llegando a reunir hasta 50 individuos residiendo en el mismo campamento.

\section{La producción material de los niños}

Los niños Nukak forman una parte significativa de los campamentos residenciales. En un estudio detallado de una muestra de 357 individuos (lo que representa un alto procentaje de la población total de los Nukak), se determinó que el 37,00\% eran menores de 10 años y este procentaje aumenta a $49 \%$ si se consideran los menores de 15 años (Franky et al. 1995: 2). Estos niños, especialmente los del primer grupo, pasan una gran parte del día dentro del campamento (Figura 2) o en su entorno inmediato (esto es frecuente en otros grupos cazadores-recolectores, por ejemplo los !Kung) (Draper 1976). En general, los niños juegan y recorren los

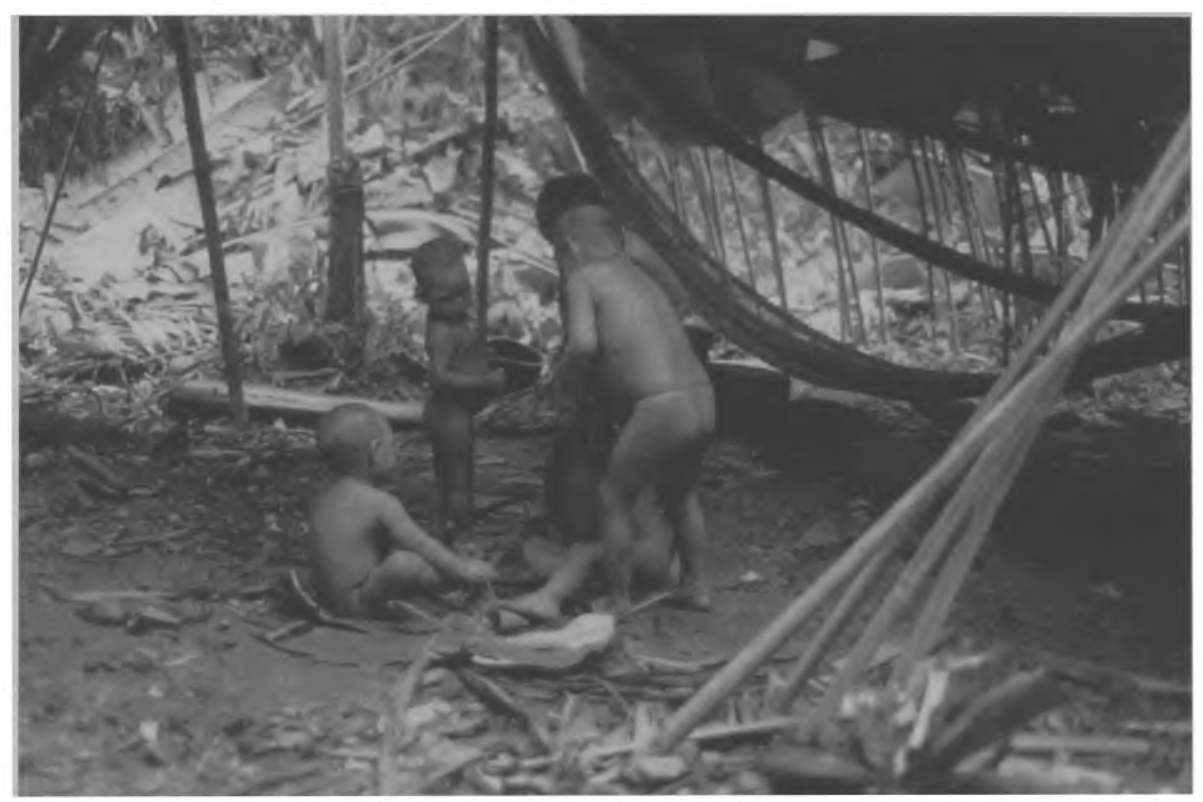

Fig. 2 - Grupo de niños Nukak jugando en la parte central de un campamento residencial de invierno. 
POLITIS, G.G. La actividad infantil en la producción del registro arqueológico de cazadores-recolectores. Rev. do Museu de Arqueologia e Etnologia, São Paulo, Suplemento 3: 263-283, 1999.

alrededores dentro de una distancia máxima establecida por la posibilidad de ser oídos desde el campamento. Debido a lo cerrado del bosque, los adultos pierden rápidamente el contacto visual con los infantes, por lo que es necesario oírlos para poder ejercer alguna vigilancia.

Se podrían reconocer tres etapas en la infancia de los Nukak, similares a otros cazadores-recolectores como los Guayakí (Clastres 1998). La primera etapa es cuando son bebés, desde que nacen hasta que caminan con cierta autonomía (aproximadamente a los 2 años de edad). Estos bebés dependen enteramente de los adultos y no generan ningún tipo de artefacto, aunque algunos objetos son fabricados para ellos (por ejemplo collares de dientes de mono y félidos, totumas, etc.) (Figura 3). Tampoco tienen aún un nombre específico y se los llama genéricamente jim 'bú o tóm'bú según el sexo. La segunda va desde los 2 hasta los 7 u 8 años. Durante este período el aprendizaje es generalizado y no hay una marcada diferencia entre los sexos. En esta etapa los niños, tanto varones como mujeres ya comienzan a ser economicamente productivos y colaboran en varias tareas de obtención de alimentos tales como la recolección o la caza y captu- ra de pequeños animales (roedores, cangrejos de río, mojojoy etc.). En muchos casos, las actividades lúdicas estan imbricadas en las tareas productivas, siendo muy difícil de separarlas entre sí o asignarle más importancia a unas que a otras. La tercera etapa es a partir de los 7 u 8 años y llega hasta la pubertad.

Los niños que acompañan a sus padres en las cacerías son los que están en la etapa final de la infancia y lo mismo ocurre con las niñas cuando salen de recolección con sus madres u otras mujeres adultas del grupo. Sin embargo la participación de ambos en las salidas de adultos es un proceso progresivo desde muy pequeños. A medida que van creciendo aumenta correlativamente la frecuencia de sus salidas acompañando a los adultos, la distancia que recorren y la participación efectiva en tareas extractivas y productivas. El único punto de inflexión se podría marcar tentativamente a los 7 u 8 años, ya que a partir de esa edad se observa un acelerado proceso de preparación específica para la vida adulta. Durante este período se nota que el proceso de aprendizaje no está basado en la imitación o la enseñanza generalizada de padres a hijos, sino en una actitud de enseñanza dirigida hacia tareas específicas de cada sexo. Por ejemplo,

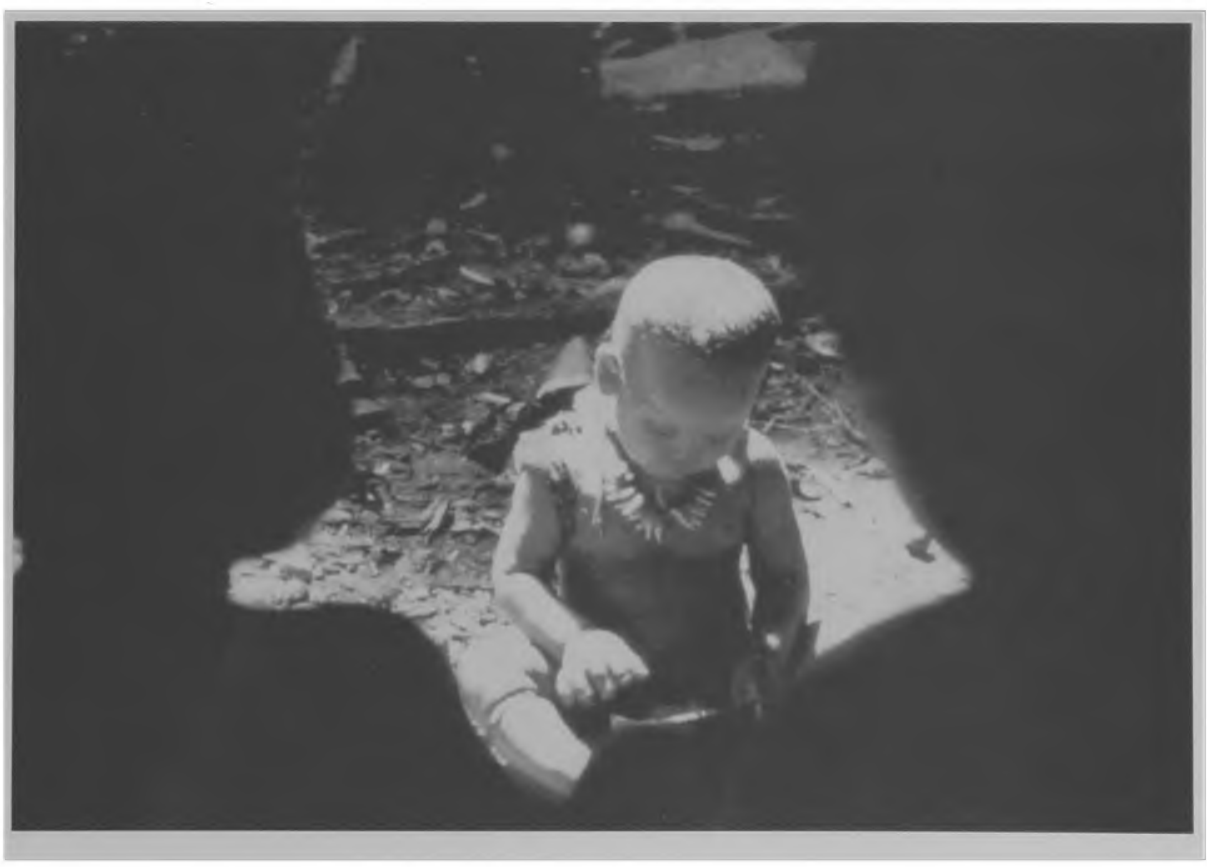

Fig. 3 - Bebé Nukak con un collar de dientes de mono, jugando con una totuma y con una cria de mono, ante la mirada de otros niños más grandes. 
POLITIS, G.G. La actividad infantil en la producción del registro arqueológico de cazadores-recolectores. Rev. do Museu de Arqueologia e Etnologia, São Paulo, Suplemento 3: 263-283, 1999.

cuando lo niños tienen alrededor de 8 o 9 años los hombres salen con sus hijos de caminata, con dardos y cerbatanas y dejan que estos prueben puntería con aves y animales pequeños. También los incentivan para que suban a las palmeras a recoger frutos e invierten algún tiempo ayudándoles a preparar cerbatanas. Alrededor de los 10 años las niñas ya cargan con frecuencia a sus hermanos menores durante los desplazamientos y casi siempre llevan un canasto cargado con enseres. Aproximadamente a partir de esta edad empiezan a despostar los monos (una activdad exclusivamente femenina) o a colaborar en esta tarea con sus madres o niñas mayores. También practican la fabricación de pulseras de fibra ( $\left.k d n^{\prime} y i i\right)$, de cestas y de burup (Figuras 4 y 5 ).

A pesar de que en la última parte de la infancia los niños pueden pasar una parte del día a cierta distancia del campamento residencial, el mayor tiempo transcurre dentro de éste y en su entorno inmediato. Cuando los niños tienen menos de 2 años, siempre están cerca de las madres y éstas los llevan en las salidas afuera del campamento para recolectar, cosechar o pescar. Después de los 2 o 3 años y en la primera parte de la infancia pueden quedarse en el campamento bajo la vigilancia de niños mayores o púberes o acompañar a sus madres. Los hermanos mayores juegan un rol importante en el cuidado de los más pequeños y pasan gran parte del tiempo a cargo de ellos. A partir de los 6 o 7 años, los niños pueden hacer algunos recorridos cortos cercanos al campamento, acompañados por niños mayores, durante los cuales recolectan algunos frutos accesibles, pescan y recogen cangrejos en charcas y arroyos de los alrededores $o$, simplemente, juegan y se divierten.

A pesar de estas salidas, solos o con sus madres, los niños pasan la mayor parte de las $12 \mathrm{hs}$. diurnas, y todas las nocturas, dentro o en las inmediaciones del campamento residencial. En éste lugar, usan y, eventualmente fabrican, tres clases de juguetes:

Clase 1: Artefactos específicamente confeccionados para jugar que tienen un diseño específico.

Clase 2: Artefactos que copian la forma de los de adultos pero que tienen un tamaño menor y que son usados en funciones similares a los de adulto o con fines lúdicos.

Clase 3: Artefactos de los adultos, enteros o

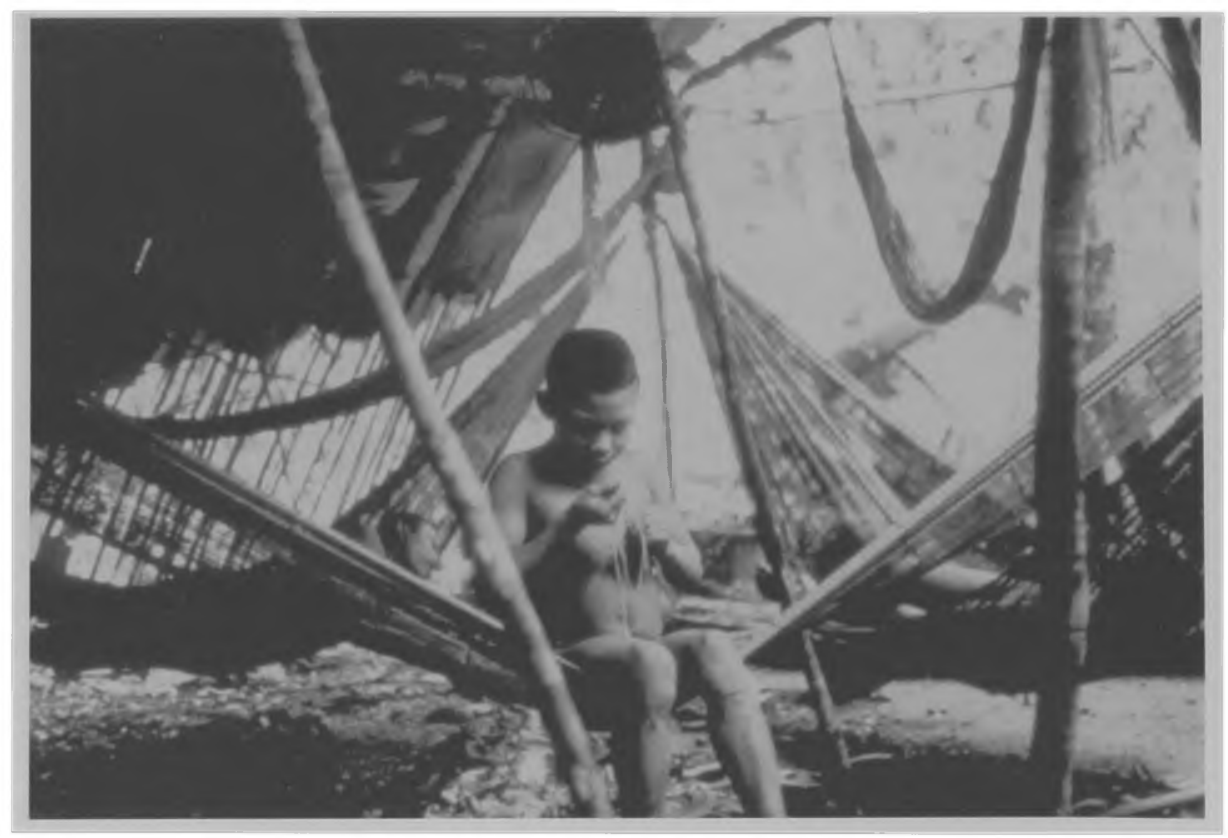

Fig. 4 - Niña Nukak tejiendo una pulsera k'dnyii dentro de un campamento residencial de invierno. 
POLITIS, G.G. La actividad infantil en la producción del registro arqueológico de cazadores-recolectores. Rev. do Museu de Arqueologia e Etnologia, São Paulo, Suplemento 3: 263-283, 1999.

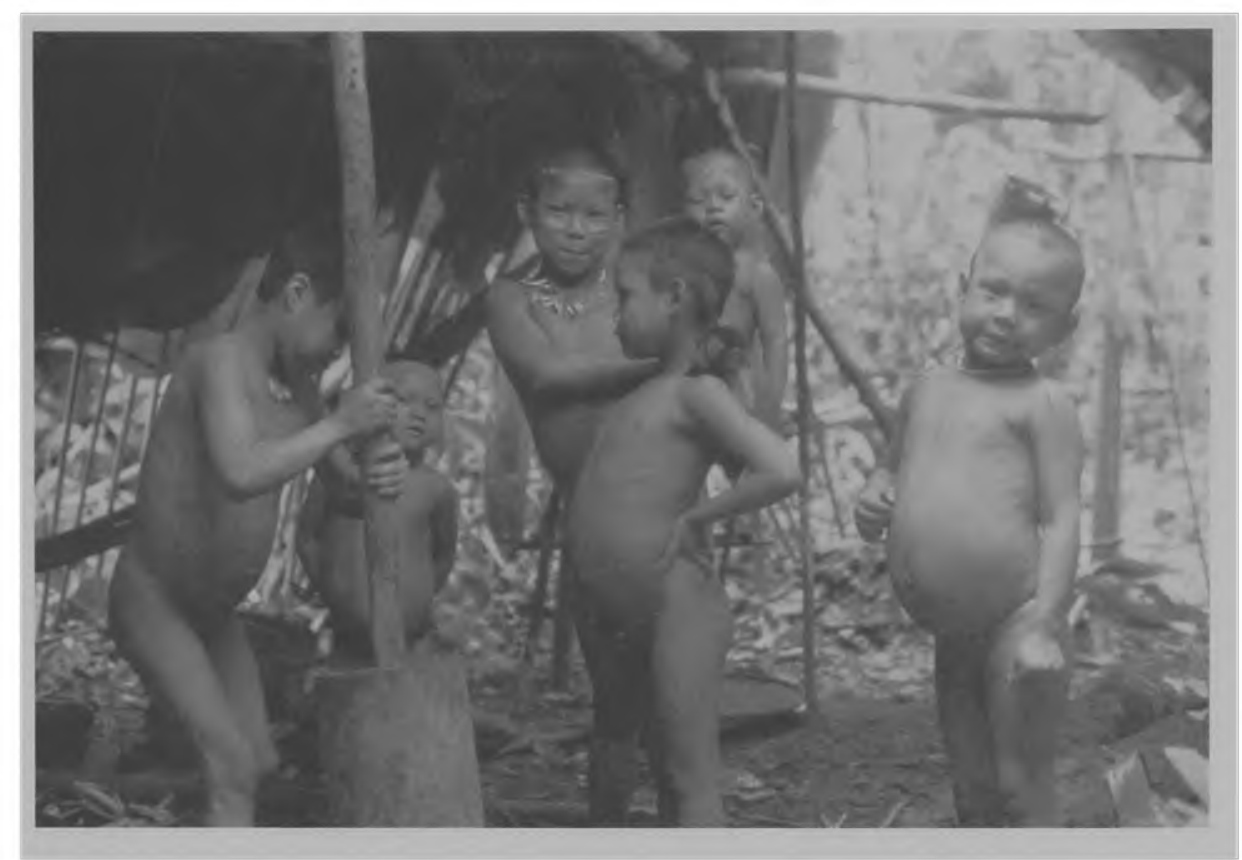

Fig. 5 - Niñas y niños Nukak machacando frutos en un mortero de madera, dentro del campamento residencial.

fragmentados, que son utilizados con fines lúdicos.

Dentro de la clase 1 se encuentran los siguientes juguetes: los aros de bejucos, los trompos de frutos, columpios de corteza o fibras y los cantos rodados. Estos no tienen artefactos homólogos entre los adultos y son fabricados por los mismos niños (a veces con la ayuda de sus padres) con modificaciones menores o incluso (como los cantos rodados) sin ningún proceso de formatización. Este sería, en nuestros términos, juguetes propiamente dichos. Pueden incluir objetos muy pequeños, como los trompos de frutos, hasta otros de tamaño mediano como los aros de bejuco de aproximadamente un metro de diámetro. Dentro de este grupo es interesante mencionar los guijarros que en algunas oportunidades los niños Nukak traen desde el "Cerro de las Cerbatanas" cuando toda la banda se acerca a estas sierras para recolectar cañas para cerbatanas (Politis 1996b: 284). Estos rodados, que sacan del lecho de los arroyos que bajan de la formación rocosa, son trasladados de un lugar a otro durante semanas o meses, hasta que finalmente se van abandonando o perdiendo en los campamentos o en sus alrededores. Los guijarros son usados exclusivamente por los niños para jugar y no se ha observado ninguna otra función.

La clase 2 contiene muchos más juguetes e incluye prácticamente todos los instrumentos hechos por adultos, pero a escala menor. Entre éstos se destacan: cestas, cerbatanas (Figura 6) dardos (Figura 7), arcos (Figura 8), flechas, balayes (cestas planas de trama más abierta), burup, totumos (recipientes de calabaza, de varias especies), arcos, vasijas de alfarería y lanzas. En esta segunda clase hay que hacer una distinción importante. Por un lado algunos artefactos son hechos por adultos, en tamaño más pequeño, para que sean utilizados por los niños, cumpliendo una función similar a la de los mayores. La única diferencia entre los artefactos de los adultos y de los niños son las dimensiones, que se adecúan a la edad y el tamaño del niño, pero la calidad de confección y la función son las mismas (por ejemplo totumos y recipientes de alfarería). Por otro lado están las réplicas de los instrumentos de los mayores, hechos por los mismos niños o sus padres, para jugar o practicar. Estos no son usados con la misma función que le dan los adultos, aunque a veces se aproxima, y la calidad de confección es menor. Esta baja calidad se debe a dos razones: a) cuando los hacen los adultos, la tecnología tiene un carácter expeditivo, debido al fin lúdico de los artefactos y no los confeccionan con el mismo 
POLITIS, G.G. La actividad infantil en la producción del registro arqueológico de cazadores-recolectores. Rev. do Museu de Arqueologia e Etnologia, São Paulo, Suplemento 3: 263-283, 1999.

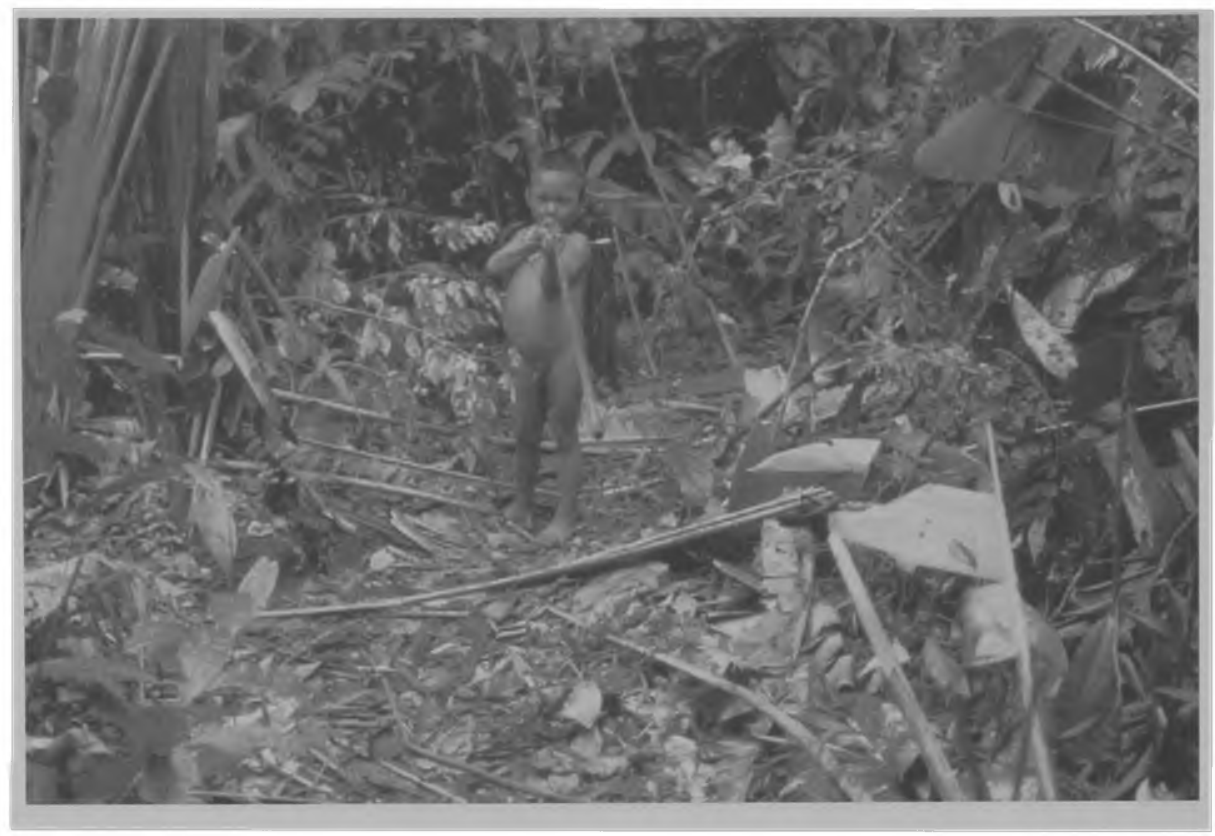

Fig. 6 - Niño Nukak jugando con una cerbatana en los alrededores del campamento.

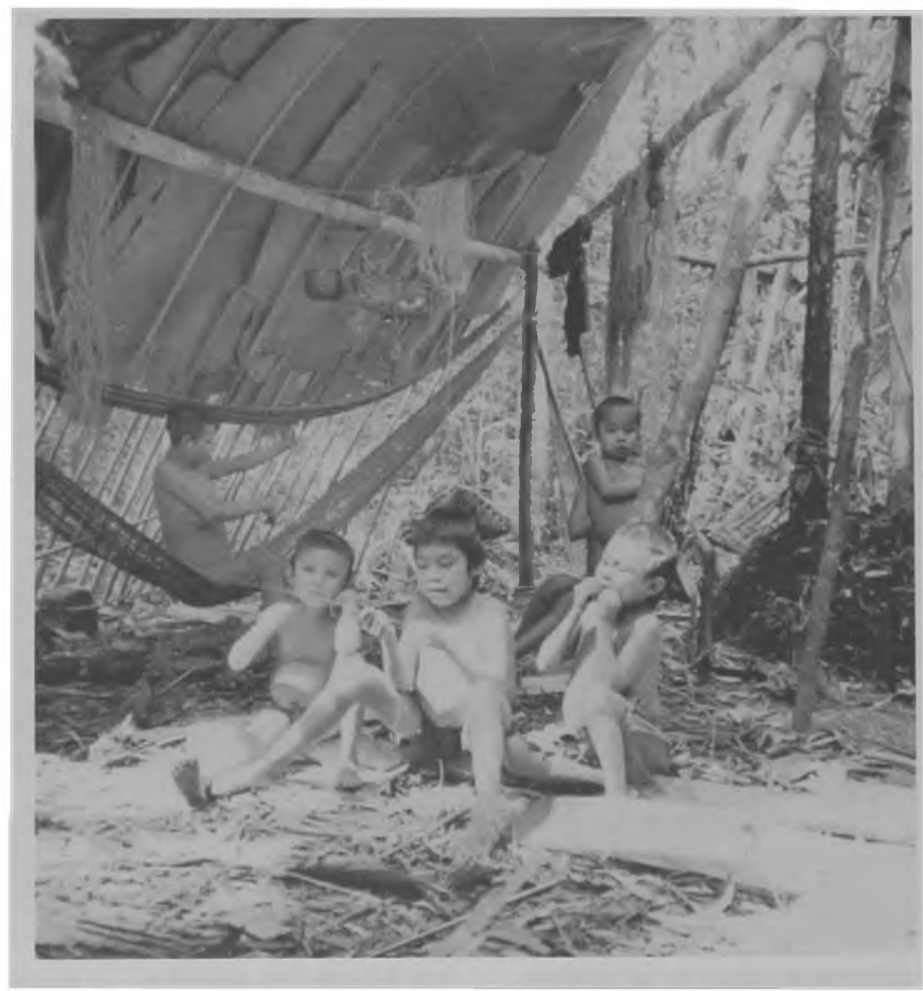

Fig. 7 - Grupo de niños Nukak fabricando dardos para cerbatana en el lugar central de un campamento residencial. 
POLITIS, G.G. La actividad infantil en la producción del registro arqueológico de cazadores-recolectores. Rev. do Museu de Arqueologia e Etnologia, São Paulo, Suplemento 3: 263-283, 1999.

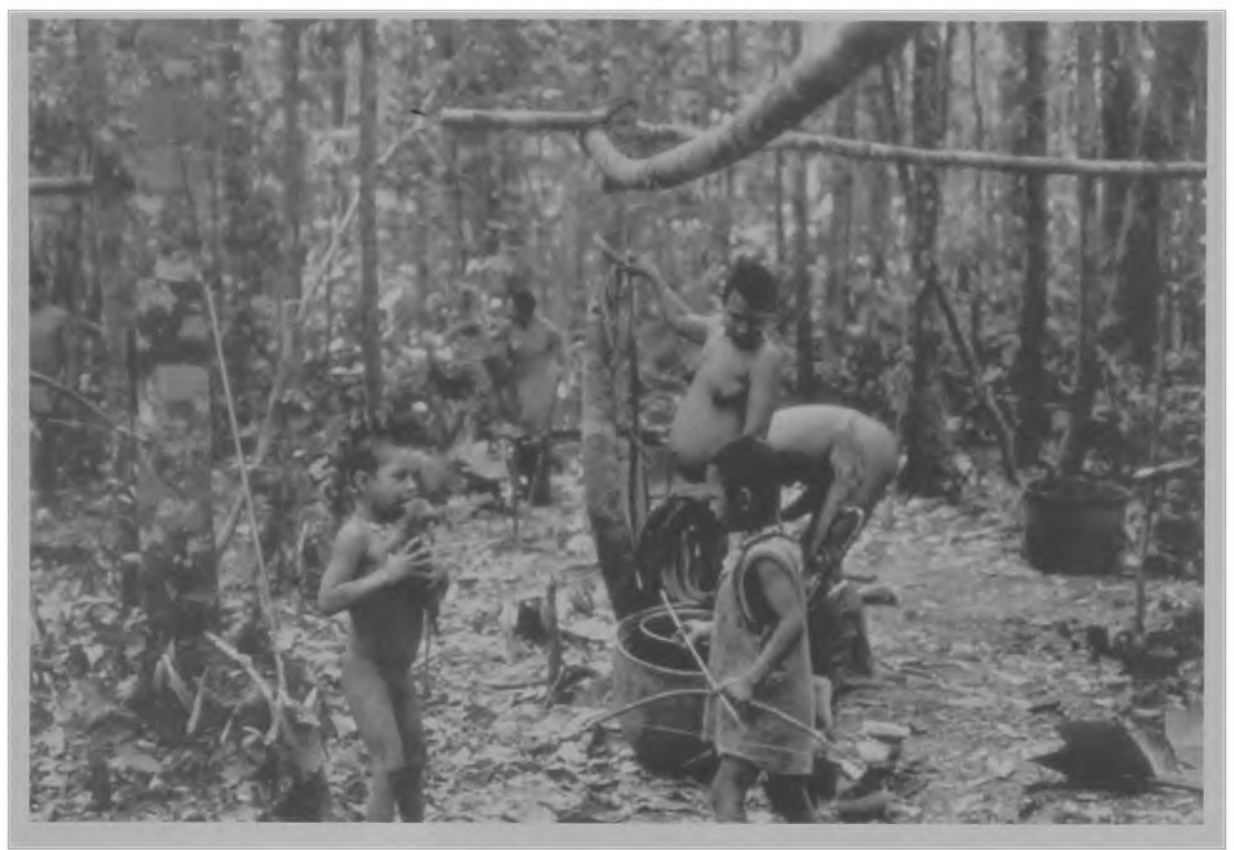

Fig. 8 - Niños Nukak jugando con pequeños arcos y flechas, mientras los adultos estan juntando las pertenencias para abandonar un campamento residencial de verano.

esmero; b) cuando los hacen los niños, la baja calidad se debe a sus limitaciones técnicas (Figura 9).

El tamaño de estos artefactos está en relación al del niño y virtualmente, dentro de un rango determinado, pueden existir todas las medidas posibles. En el caso de las cerbatanas, por ejemplo, las más pequeñas que registramos era de $0.82 \mathrm{~m}$ de longitud. De allí en adelante hay una gradación hasta la de los adultos que llegan a los $3.20 \mathrm{~m}$. La diferencia entre la de los adultos y la de los niños se dá por la relación que existe entre el largo de la cerbatana y la estatura y habilidad del usuario.

La clase 3 esta formada por artefactos de adultos, enteros o rotos, que son usados por los niños como juguetes de manera ocasional (Figuras $10 \mathrm{y}$ 11). Estos objetos no son modificados y la actividad de los infantes afecta sólo a su distribución espacial. Esta clase es la más utilizada por los más pequeños, que toman como juguete cualquier objeto que tengan cerca.

Los artefactos de los niños también se diferencian de los de adultos por el locus de descarte. En el primer caso, los niños descartan la inmensa mayoría de sus objetos en el campamento residencial o en sus inmediaciones, mientras que los adul- tos también lo hacen lejos de los campamentos donde realizan múltiples actividades (cacería, recolección, despostamiento de pecarí, etc.). Uno de los casos más interesantes es el de los dardos ya que los niños juegan con mucha frecuencia con cerbatanas y hacen prácticas de puntería dentro del campamento. Como consecuencia de esto, en el piso de los campamentos abandonados quedan abundantes dardos pequeños, a veces enteros, como producto de esta actividad.

En algunas ocasiones, los niños construyen pequeñas viviendas dentro del campamento, que son una réplica en miniatura de las viviendas familares de la estación lluviosa. Otras veces construyen estas pequeñas réplicas en los alrededores de los campamentos residenciales y allí pasan el dia jungando e imitando a los adultos. En estos pequeños campamentos quedan entonces una serie de objetos pequeños y generalmente de mala calidad, y también se encuentran uno o dos fogones pequeños.

\section{La producción material de los niños en otros grupos cazadores-recolectores americanos}

La generación de varias clases de artefactos 
POLITIS, G.G. La actividad infantil en la producción del registro arqueológico de cazadores-recolectores. Rev. do Museu de Arqueologia e Etnologia, São Paulo, Suplemento 3: 263-283, 1999.

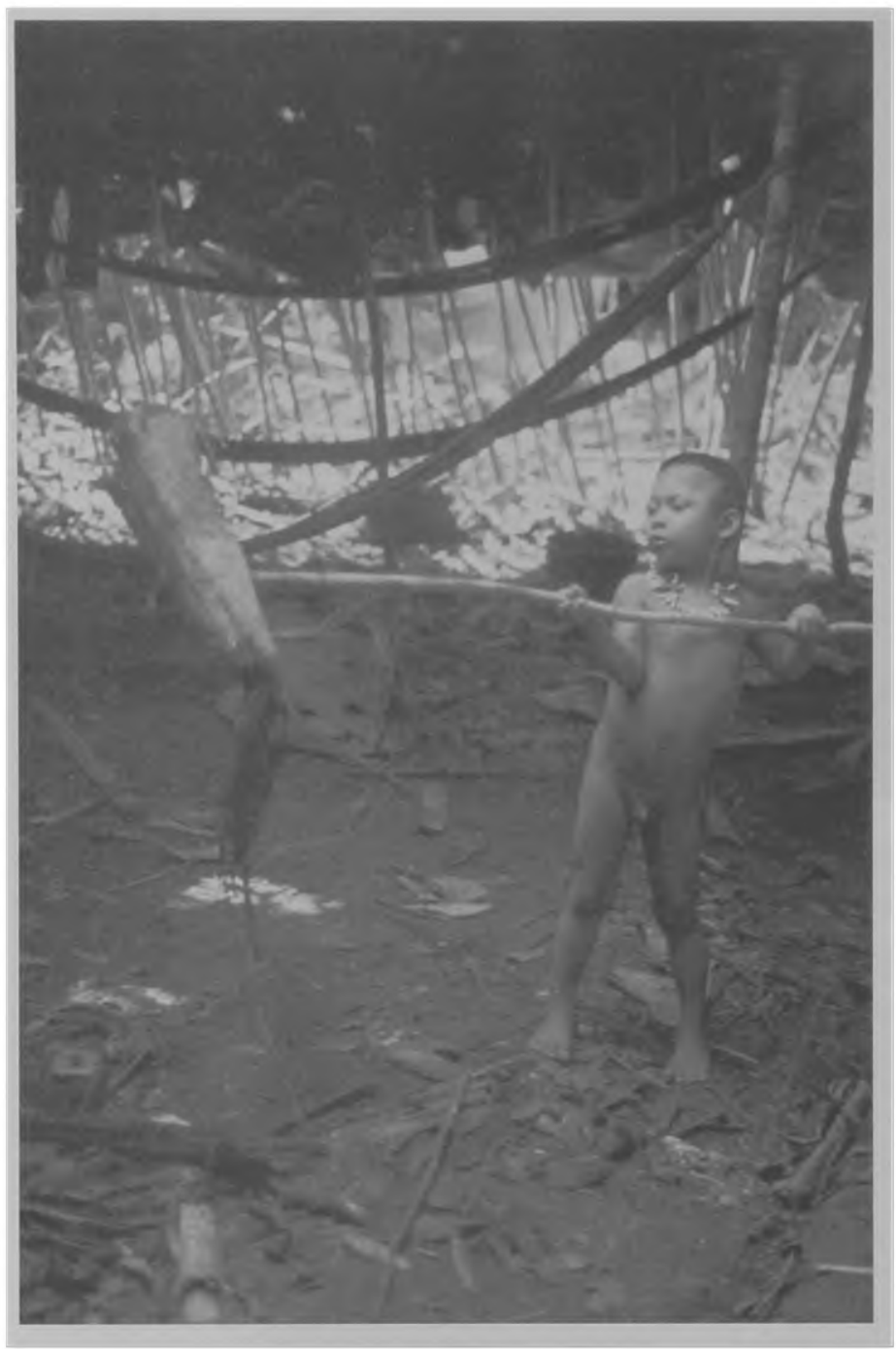

Fig. 9 - Niño Nukak practicando dentro de un campamento con una lanza expeditiva hecha por el mismo.

hechos por o para los niños es una conducta universal que se ha registrado en todos los grupos de cazadores-recolectores estudiados desde un punto de vista antropológico. Se han obtenido datos interesantes tanto en crónicas y documentos históricos como en trabajos etnográficos. Un rápido resumen de algunos ejemplos permitirá visualizar tendencias generales y quitarle particularismo al caso Nukak. Además, algunos ejemplos se refieren directamente a objetos que son muy comunes en los sitios arqueológicos de América del Sur, tales como puntas de proyectil, bolas de boleadora, etc.

Para el caso de las puntas de proyectil, las citas inventariadas por Dawe (1997) para América del Norte son particularmente interesantes. Entre éstas se destaca la de Grinnell (1923) quien observó que tan pronto un niño Cheyenne "was able to run easily, a small bow and some arrows were made for him"; también la de Schoolcraft que indica: "Boys were always furnished with small arrow-points" (Schoolcraft 1851 en Dawe 1997: 307) o los comentarios de Wallace y Hoebel (1952:16) que indican que entre los Comanches históricos los padres comenzaban a enseñarle a sus hijos varones a fabricar el equipo de cacería (incluyendo las puntas de proyectil) a la edad de 5 años. Dawe (1997) ha demostrado como entre diversos grupos indígenas de las llanuras la confección de puntas de proyectil pequeñas era frecuente para que los niños jugaran y adquirieran las habilidades para la caza. Estas citas coinciden con lo observado entre los Nukak, en cuanto al tamaño menor del equipo y a la existencia de homólogos entre los adultos. Hay también otras similitudes, como lo expresa la primera cita, en cuanto a que las puntas eran hechas para los niños por los adultos (como algunos artefactos Nukak) y que hay un relación entre el tamaño del artefacto y la estatura y edad del niño. Entre los grupos de la cuenca inferior del río Mississippi, Swanton mencionó que cuando los niños “....approach 12 years, a bow and arrow are made for them, proportioned to their sthrengh (Swanson 1911 en Dawe 1997:307). En este sentido, se ha observado que las flechas y los arcos eran construidos dependiendo de la altura del arquero (Bourke 1891) y por lo tanto en el registro arqueológico es esperable encontrar un continuum de tamaños de puntas de proyectil. Este rango podría variar desde "small points enough to fit a two years old boy's arrow, to those having the optimum dimensions of those for adult weaponery" (Dawe 1997). En el caso de las cerbatanas, dardos, arcos y flechas Nukak la 
POLITIS, G.G. La actividad infantil en la producción del registro arqueológico de cazadores-recolectores. Rev. do Museu de Arqueologia e Etnologia, São Paulo, Suplemento 3: 263-283, 1999.

situación es la misma.

Entre los Yámanas de los Canales Fueguinos, Gusinde (1987) relata con mucho detalle las actividades de los niños y los objetos que utilizan. En una cita menciona que no hay juguetes propiamente dichos para los varones (los que en este trabajo se incluyen en la clase 1) y destaca la manufactura de objetos que pueden ser claramente incluidos en la clase 2: "No existe un juego propiamente dicho para los chicos. Un adulto, generalmente el padre, les construye reproducciones de armas y utensilios, de que se sirven los hombres para cazar... Naturalmente estas piezas se arruinan pronto o se pierden, pero infatigablemente los adultos construyen un sustituto, y cuanto más crece el niño tanto más grande son tales utensilios" (Gusinde 1987: 729). Este autor relata que estas reproducciones de armas no son muy efectivas y que "apenas sirven como juguete" ya que no se les da a los niños un arma completa con la punta de hueso pues esto sería demasiado valioso. Como alternativa "se usan varas rectas, cortadas en punta o astas de arpón inútiles" (Gusinde 1987: 729).

El ejemplo de las niñas Yámanas es más complejo pues sí existen juguetes propiamente dichos

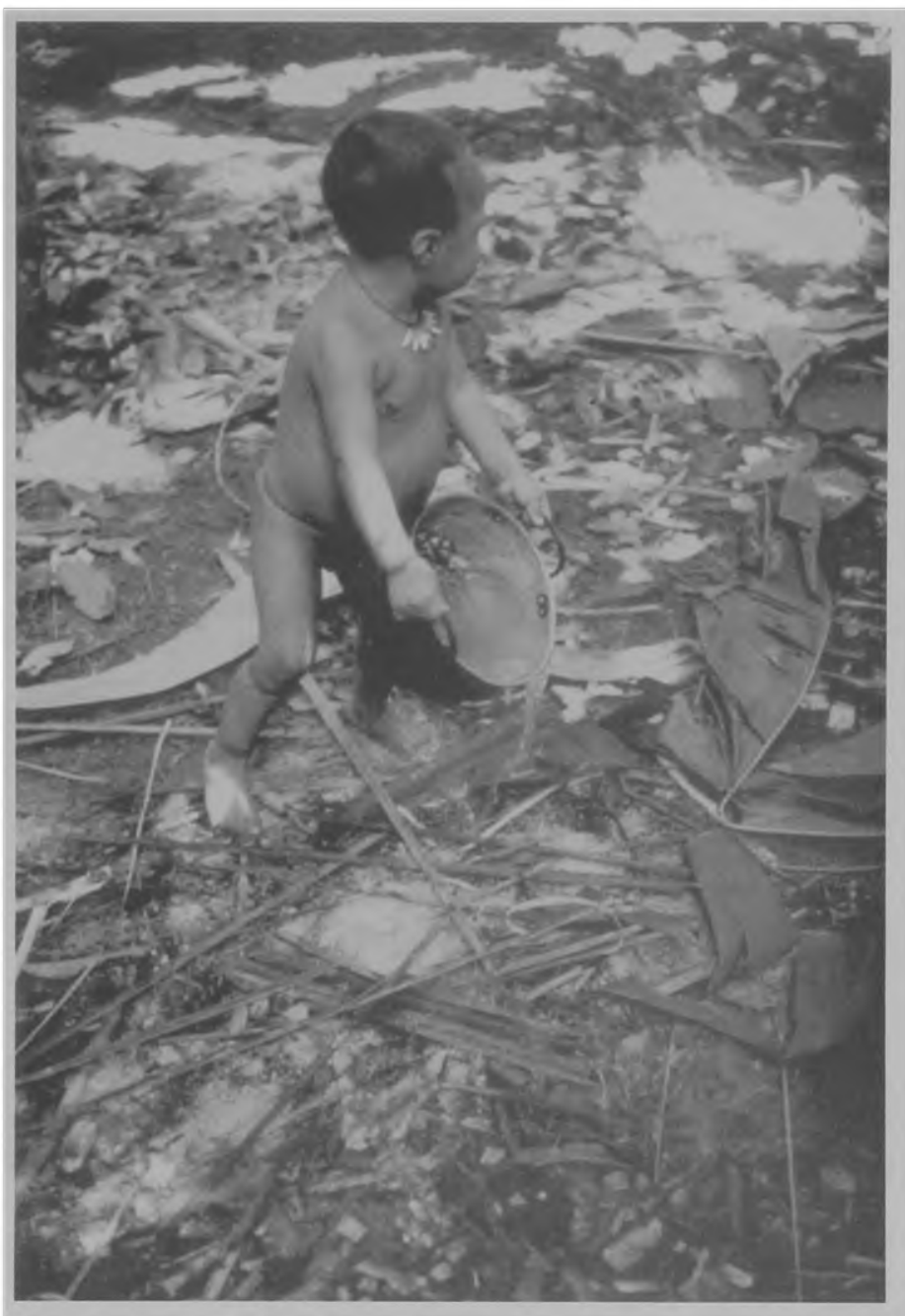

Fig. 10 - Niño Nukak volcando el agua de una olla de metal dentro del campamento residencial. tales como unas muñecas muy simples hechas con una piedra cilíndrica y un fragmento de madera (Gusinde 1987). Las niñas parecen ser económicamente más activas que los varones y desde pequeñas fabrican raspadores, trenzan canastos, arman cordones para adornos etc. Además, como el manejo de la canoa es una responsabilidad femenina, desde muy pequeñas son entrenadas en ésto mediante la construcción de pequeñas canoas acordes con su tamaño y edad: “ ... el padre construye un pequeña reproducción de esta canoa para su hija semiadolecente. Concuerda en todos sus detalles con una canoa grande y sus medidas son tales que permiten a una sóla niña sentarse dentro y pasear con ella" (Gusinde 1987: 733).

Entre los Selknam también existe una rica literatura que describe los juegos infantiles y los objetos que usan los niños. Gallardo (1910) menciona a las puntas de proyectil pequeñas como jueguetes infantiles, utilizadas sólo para diversión y entrenamiento. Gusinde (1982: 373) describe la intensa actividad de las niñas dentro de los campamentos: "Las niñas pequeñas se ejercitan con todo lo que le cae en las manos. Hay un continuo palpar y tirar toda clase de objetos, coger bichos y plantitas, tirar de correas, golpear madera, tirar de trozos de piel, rizar la lana del propio vestidito, chocar contra un objeto erguido" Este autor tam- 
POLITIS, G.G. La actividad infantil en la producción del registro arqueológico de cazadores-recolectores. Rev. do Museu de Arqueologia e Etnologia, São Paulo, Suplemento 3: 263-283, 1999.

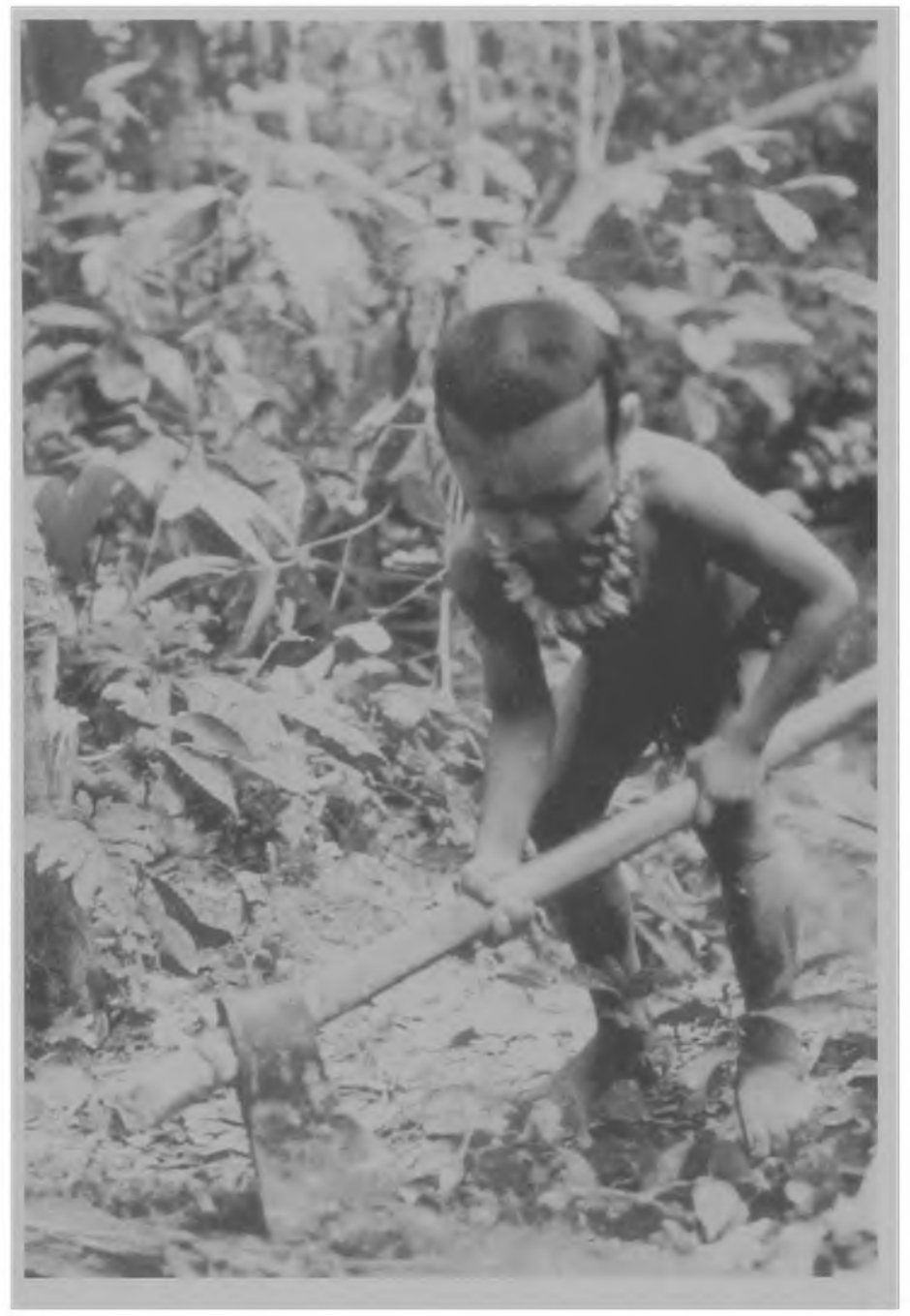

Fig. 11 - Niño Nukak julgando con un hacha en los bordes del campamento residencial.

bién describe la elaboración muy cuidadosa y compleja de unas muñecas que requieren de mucho trabajo y dedicación. Una de las citas más interesantes es la que se refiere a la confección de arcos para chicos: "El padre ya los prepara para el lactante en la forma más simple posible: un tosco palito se dobla levemente y se mantiene en esta curvatura mediante un hilo de tendón, una varita superficialmente raspada sirve de flecha. Aunque el niñito por el momento sólo es capaz de zamarrearlo y tirar de él, los padres tienen que verlo en sus manitas, como si, de lo contrario, le faltara algo. He visto tales arcos de sólo doce centímetros. A medida que el niñito crece, crecen también sus juguetes. Como en el cuarto o quinto año de vida éstos dejan de ser un mero pasatiempo, en el futuro el padre realmente los hace con mucho cuidado. De ahora en adelante son una imitación del arco de caza propiamente dicho" (Gusinde 1982: 376-377). Un caso similar pasa con la honda ya que "a pesar de su tamaño más pequeño se asemeja exactamente a la del hombre" (Gusinde 1982: 378).

Entre los Tehuelches septentrionales de la Patagonia, Musters describió a fines del siglo pasado en varios pasajes de su libro como eran algunos artefactos usados por los niños. Uno de estos párrafos narra que: "Las criaturas tenían varias [crías de ñandú] para diversión, acostumbraban soltarlos para cazarlos con boleadoras diminutas, lo que por lo general acababa con la muerte de ellos" (Musters 1997: 176). Otro de los párrafos explica muy claramente las actividades de los niños, las que son muy similares a las registradas entre los Nukak: "Las criaturas se entretienen en general en imitar a las personas mayores: los muchachos juegan con boleadoras diminutas y cazan perros con pequeños lazos, y las muchachas construyen tolditos para sentarse dentro de ellos, y con todo este objeto unos y otros se llevan sin que nadie los reprima, todo lo que les parece conveniente" (Musters 1997: 205). Otra cita hace referencia a un entretenimiento de los muchachos con piedritas y parecido al juego infantil de la época conocido como el "juego de tabas " En esta citas se identifican los objetos asignados a las categorías 1,2 y 3 ya definidas en este trabajo. Musters también explica que era raro que los hijos varones salgan de cacería con sus padres antes de tener 10 o 12 años y que recién participaban en los combates a los 16 años; las niñas ayudaban en las tareas domésticas y en la fabricación de objetos a 
la edad de 9 o 10 años y a los 16 años ya eran consideradas aptas para el casamiento (Musters 1997: 210).

Entre los Guayaquí de Paraguay, Clastres (1998) ha recogido interesantes datos sobre la actividad infantil entre cazadores-recolectores. En principio también reconoce tres etapas de la infancia. La primera hasta los 3 años, cuando son lactantes y pasan casi todo el tiempo al lado de su madre. La segunda llega hasta los 7, y la tercera hasta los 15 años aproximadamente. En esta última etapa, que se denomina kybuchu y los varones ya tienen su set de arcos y flechas que fueron regalados por algún adulto (que no siempre es su padre). Dentro de este grupo de edad "... boys were already well trained in handling their weapon; without going far away from the camp, they could spend hours alone in the woods stalking prey suitable to the power of their bows (which was not negligible)" (Clastres 1998: 96).

Entre los Sirionó del oriente de Bolivia, Holmberg (1978) ha registrado también un patrón similar al de los Nukak en lo referente a las armas infantiles. Este autor expresa que antes de que un niño cumpla los 3 años su padre ya le ha hecho un arco y flechas en miniatura y que aunque no puedan ser usados por el niño durante varios años, simbolizan su papel como cazador. A partir de los 3 años los niños ya estan usando alguna clase de arco y pasan muchas horas jugando y disparando flechas a cualquier blanco inerte. Ya más grandes disparan contra mariposas y pájaros y para la edad de 8 años ya han cazado algún animal pequeño. Para el caso de las niñas, antes de que cumplan 3 años sus padre les han hecho una pequeña rueca para que practiquen más adelante el arte de hilar. En general, la mayoría de los juguetes caen dentro de la clase 2 , ya que replican los instrumentos de los adultos: "En forma algo sorprendente, los arcos y flechas en miniaturas para los muchachos y las ruecas para las chicas, son los únicos juguetes que los Sirionó hacen para sus hijos"'(Holmberg 1978: 186).

En suma, las observaciones sobre los grupos indígenas de las llanuras norteamericanas y los de América del Sur, permiten identificar las tres mismas clases de artefactos infantiles que las reconocidas entre los Nukak. En éstos casos también se pueden identificar las dos variantes de objetos de la clase 2 , es decir, los confeccionados por los propios niños y los que manufacturan para ellos los adultos, generalmente los padres, y que son habitualmente de mejor calidad. Además se destaca la similitud de la correlación entre el tamaño del artefacto y el del usuario. En los casos norteamericanos y en el de los Yámanas, Selknam y Sirionó esto se observa para los arcos y flechas, en el caso Nukak la relación es entre las armas de caza y pesca como las cerbatanas, dardos, lanzas y arcos.

\section{Expectativas arqueológicas}

Es posible generar expectativas arqueológicas para reconocer por lo menos dos de las tres clases de artefactos infantiles dentro del registro arqueológico. En principio, la clase 3 es indeferenciable, en cuanto a morfología, tecnología y dimensiones, pero las otras dos tienen características que permiten su identificación dentro de un contexto arqueológico (Tabla 1).

La otra forma de identificación, que debe ser analizada en conjunto con las características precedentes, es el lugar de descarte. Mientras que los artefactos de la clase 1 son descartados exclusivamente donde fueron usados para actividades lúdicas, en la clase 2 los objetos son desechados también donde fueron usados como juguetes, lo que en muchos casos son diferentes al lugar donde se abandonan los homólogos adultos. Esto es notable en el caso de los dardos, las flechas las lanzas y otros objetos de hombres adultos que son abandonados (usualmente rotos) en sitios afuera y a cierta distancia de los campamentos residenciales, donde se llevó a cabo la cacería. En artefactos tales como vasijas, cestas, balayes etc., tantos los de adultos como los de niños son descartados en el campamento residencial. Obviamente en to-

TABLA 1

\begin{tabular}{|c|c|c|c|c|}
\hline \multicolumn{5}{|c|}{ Diferencia entre artefactos infantiles en relación con los de los adultos } \\
\hline & Homólogos & Dimensiones & Tecnologia & Locus de descarte \\
\hline Classe 1 & no & variable & $\begin{array}{l}\text { baja calidad o } \\
\text { sin modificación }\end{array}$ & co-varia con función \\
\hline Classe 2 & si & menor & menor calidad & $\begin{array}{l}\text { no co-varia con función } \\
\text { (en algunos casos) }\end{array}$ \\
\hline
\end{tabular}


dos los casos, el lugar de descarte inicial puede cambiar debido a las actividades de limpieza del campamento (Politis en prensa).

En ciertos contextos hay algunos artefactos de adulto que podrían ser confundidos con instrumentos infantiles: a) elementos de diseño pequeños, b) elementos que se reducen por uso, y c) miniaturas o réplicas a escala menor. En los tres casos, en principo, habría algunos indicadores que permitirían discriminarlos de los artefactos infantiles. En el primer caso, los artefactos pequeños de adultos (por ejemplo puntas de proyectil) tienen una buena calidad de confección y se encontrarían en loci de descarte relacionados con la función que tuvieron (por ejemplos loci de cacería). Además habría que analizar el conjunto arqueológico completo y en la mayoría de los casos no se hallarían homólogos más grandes (como sí sucede en los artefacto infantiles).

En el segundo caso también es posible distinguir entre el tamaño pequeño de artefactos infantiles y la disminución debido al uso. En general, la reducción por uso esta acompañada de otros rasgos que permiten identificar la intensidad de uso (por ejemplo filos muy abruptos en el material lítico, o reducción del largo de las cerbatanas). Un ejemplo interesante es el de los porotadí, unos artefactos de madera alargados con un extremo biselado que son usados por los Ayoreo orientales para raspar la pulpa de una raíz comestible (Bórmida 1973). Cuando este artefacto se va desgastando, el filo se reaviva y va perdiendo entonces longitud hasta llegar a los dos tercios del largo original. Por las connotaciones míticas que tiene (además es usualmente decorado con signos clánicos) cuando llega a este grado de desgaste, se dice que "envejeció" y sólo lo pueden usar los viejos mientras que los jóvenes deben tener cuidado en no transportarlo en las bolsas que llevan en la espalda (Bórmida 1973: 50-60). Este ejemplo permite anticipar que el caso del porotadí no sería identificado, siguiendo la metodología propuesta, como un artefacto infantil a pesar de existir dentro del mismo contexto ejemplares de variado tamaño. Por un lado, es una sola dimensión la que se reduce, el largo, mientras que las otras dos se mantienen constantes. En éste sentido, los porotadí "envejecidos" no son más pequeños, sino sólo más cortos. Por otro lado, la buena manufactura de los más usados, incluyendo incisiones que los connotan clánicamente, los permitiría separar de artefactos infantiles de la clase 2.
El tercer caso es quizás el más difícil de discriminar. Un buen ejemplo es el de las miniaturas que usan algunos adultos Inuit. En principio se ha propuesto que muchas de las miniaturas Inuit serían artefactos infantiles y que estos objetos representan el status de los niños que son considerados como "adultos pequeños" (Park 1998). La forma de diferenciar las miniaturas infantiles de las que se ponen en los entierros de algunas personas y las que usan los shamanes es aún problemática, sobre todo por la buena calidad con que estan hechas las primeras. Sin embargo, esto sería posible ya que los tres tipos de miniaturas tienen loci de descarte distintos, y en un caso (el de los entierros de adultos) esto sería lo suficientemente informativo como para no adjudicarlas, en el contexto de la cultura Inuit, a artefactos infantiles.

\section{Conclusiones}

En este trabajo se ha intentado contribuir a generar una metodología que permita identificar la actividad de los niños en los depósitos arqueológicos. Este es un primer paso para otro objetivo mas ambicioso: explorar la agencia infantil en las sociedades cazadoras-recolectoras del pasado. Los niños forman porcentajes significativos de las poblaciones humanas, y además pasan mucho tiempo en los campamentos residenciales. Esto tiene varias implicancias fuertes para la arqueología. La primera es que en estos campamentos los desechos infantiles van a ser significativos. La segunda es que la identificación de contextos producidos por niños en diferentes etapas de la infancia, sería un indicador complementario para proponer la tendencia funcional de un sitio como un campamento residencial. La tercera, es que los conjuntos discretos de artefactos infantiles de la última etapa de la infancia podrían ser un buen indicador de la función de los sitios de actividades específicas (por ejemplo sitios de caza y procesamiento) y permitirían reconstruir más adecuadamente las tareas desarrolladas en el lugar.

Teniendo en cuenta el primer enunciado, se debe esperar entonces que los niños produzcan dentro de los campamentos residuos. Estos residuos abarcaran una amplia gama de desechos que no estan en función de una cadena operativa destinada a la producción de artefactos para ser usados en tareas tecno-económicas o como items de valor 
simbólico. Estos artefactos y sus desechos tendrían trayectorias diferentes que no se explicarían dentro de modelos de optimización de materia prima.

En base a la información etnográfica de cazadores-recolectores se puede anticipar que los sitios generados por este tipo de sociedades en el pasado, cuando se trate de campamentos residenciales, contendrán porcentajes significativos de artefactos producidos por los niños. Esto puede incluir desde la práctica en la reducción de nódulos, hasta el entretenimiento con lascas primarias y secundarias, y la confección de diversos artefactos generalmente de baja calidad. Lo mismo se puede esperar con la alfarería, con el entrenamiento en la decoración y con la ejercitación en la confección de vasijas. En consecuencia se debería esperar en los loci de manufactura de cerámica, masas de arcilla y recipientes pequeños moldeados por los niños que estaban junto a los adultos durante el proceso de confección.

Desde una perspectiva normativa, en la aqueología funcionó el razonamiemto de que la distancia étnica o cultural entre distintas poblaciones del pasado podría ser medida en términos del grado de similitud de sus conjuntos arqueológicos (Jones 1997: 25). La arqueología procesual alertó sobre las diferencias en la función de los asentamientos, en los modos de explotación de los recursos y en las estrategias tecnológicas como fuentes de variación arqueológica dentro de las mismas sociedades (entre muchos otros Binford 1977, 1978, 1979). Sin embargo, esta perspectiva ha derivado en cierto "tecnocentrismo" ya que ha considerado a los artefactos como simples herramientas usadas para fines estrictamente prácticos y funcionales, sin considerar otras dimensiones de la cultura material tales como su contenido simbólico. La etnografía ha demostrado como los objetos estan incluidos en sistemas simbólicos que afectan su uso, reuso y descarte (ver entre muchos ejemplos Bórmida 1973, Toth et al. 1992, Politis 1996b). El reconocimiento del amplio rango potencial de significados en la cultura material ha sido el foco de la arqueología cognitiva y post-procesual (Whitley 1998: 99). Ahora, la consideración de los grupos etarios en la formación del registro arqueológico genera perpectivas novedosas ya que esto constituye una fuente signficativa de variación artefactual, cuya dimensión utilitaria es distinta según al grupo de edad al que pertenezca el usuario.

Las actividades tecnológicas no son sólo la consecuencia de tareas planeadas, concientes y dirigidas hacia la obtención de artefactos funcionalmente eficientes. Son también el resultado de procesos de aprendizaje y de enseñanza. Entre los ancianos, la producción de objetos de determinadas maneras significa también una via para mantener el status y el prestigio. Las habilidades tecnológicas de los más viejos tiene sus particularidades, que se deberían poder reflejar en las tendencias del registro arqueológico. Tomando en cuenta estas consideraciones, se abre una nueva perspectiva en los estudios de la cultura material de los cazadores-recolectores, ya que es bastante claro que el registro producido es multidimensional y responde a una variedad de causas entre las que se incluyen, la enseñanza y el aprendizaje, la diversión y el juego, y el uso simbólico de los objetos para mantener prestigio social. ¿Qué porcentaje de los desechos han sido generado en un proceso lineal de producción de un equipo de artefactos utilitarios?, ¿Cuánto ha derivado de la acción recurrente y constante de los niños jugando y practicando dentro de los campamentos?. Esto, salvo pocas excepciones (por ejemplo Bodú et alii 1990, Nami 1994) no ha sido planteado en el estudio de la tecnología lítica de los cazadores-recolectores del pasado. Sin duda, la respuesta es difícil y distinta para cada caso de estudio, pero en principio la mejor forma de abordar el análisis sería desde una perspectiva que diera cabida a múltiples agentes, no todos tratando de usar la materia prima de manera óptima, no todos suficientemente entrenados y habilidosos, no todos preocupados por obtener artefactos útiles y eficientes en términos tecno-económicos.

El rango de variación de tamaño y calidad de algunos artefactos (especialmente las armas de caza) según la edad y la estatura del usuario tiene también implicaciones importantes para el análisis y la interpretación arqueológica. En primer término, indica que los artefactos de adultos y los de niños no se separan en dos conjuntos discretos sino que se deben visualizar como un continuum de tamaño y calidad. Dentro de este continuum existen infinitas combinaciones dependiendo, entre otros factores, de la estatura, la edad y la habilidad del fabricante o usuario. De esta manera, se puede esperar que algunos artefactos, usados frecuentemente como diagnósticos en la interpretación arqueológica, pueden variar en la forma y las dimensiones en función de estas tres propiedades. La variabilidad en el diseño de las puntas de proyectil por ejemplo, ha sido ampliamente utilizada como 
indicador de distinto tipo en la arqueología de los cazadores-recolectores (por ejemplo etnicidad, idiosincracia, jerarquía social, función, etc.). No niego que éstas u otras sean causas de la variación estilística y técnica de las puntas de proyectil u otros artefactos (i.e. Wiessner 1983), pero propongo que la variabilidad artefactual también es consecuencia del grupo de edad y que ésto debe ser tomado en cuenta, no como un elemento contingente, sino como un factor recurrente en la generación de la variabilidad de los depósitos arqueológicos.

Otro punto importante para comenzar a explorar es la participación de los niños en la reproducción de la cultura material. En varios ejemplos etnográficos (ver por ejemplo Gusinde 1982) y en el caso de los Nukak, se ha observado que algunos elementos que ya no son usados por los adultos (pero que lo fueron en el pasado cercano) continúan presentes como juguete entre los infantes. Este sería por ejemplo el caso de los arcos y las flechas que entre los Nukak los niños usan con frecuencia aunque los adultos ya han abandonado esta práctica. Este tema permitiría discutir el agente infantil dentro de la sociedad, ya que algunos elementos materiales, que ante situaciones de contactos culturales intensas dejan de ser usados por los adultos, sobreviven entre los niños. De esta manera, los infantes se transformarían en una suerte de "reservóreos" artefactuales, y mantendrían circulando objetos, que de otra forma desaparecerían de la sociedad.

Además de los niños, como se ha expresado, los ancianos hacen artefactos de diferente manera y con otros propósitos más alla de los estrictamente utilitarios. También los adolecentes fabrican objetos específicos generalmente relacionados con los rituales de pasaje. La literatura etnográfica abunda en ejemplos de estos rituales, para los cuales se confeccionan artefactos específicos. De esta manera, parece bastante claro que los grupos de edad van generando dentro de las sociedades cazadorasrecolectoras, una variedad de útiles que no estan sólo funcionando dentro de la esfera tecno-económica, sino que tienen múltiples dimensiones de significados, que estan relacionados con el aprendizaje, la diversión, el status, la enseñanza, el ritual, etc. Con una adecuada metodología sería posible ver como la cultura material va transformándose a lo largo de la vida de los individuos y como ésto va generando tendencias en depósitos arqueológicos diferentes. En el caso que trata este artículo las expectativas generadas a partir de información etnoarqueológica, etnohistórica y etnográfica no deben ser usadas como recetas de aplicación universal, sino como guías o referencias a partir de las cuales se pueda comenzar a explorar los paisajes arqueológicos formados por sociedade en las cuales los niños son activos productores y consumidores de cultura material, y en consecuencia responsables importantes en la generación de registro arqueológico.

\section{Agradecimientos}

Los trabajos de campo entre los Nukak fueron financiados por dos subsidios (nros. 5389 y 5701) de la Wenner Gren Foundation for Anthropological Research y entre 1995 y 1996 por el Instituto Amazónico de Investigaciones Científicas SINCHI (Colombia).

Esta investigación forma parte de los proyectos del programa INCUAPA (Núcleo de Investigaciones Arqueológicas y Paleontológicas del Cuaternario Pampeano) de la Universidad Nacional de Centro de la Pcia. de Buenos Aires. Este programa recibe subsidios de la Universidad Nacional del Centro de la Pcia. de Buenos Aires, de la Agencia Nacional de Promoción Científica y del CONICET de la República Argentina.

\section{Bibliografía}

ARDILA, G.

1992 Los Nukak-Makú del Guaviare: mi primer encuentro con la gente de las palmas (etnografía para la arqueología del poblamiento de América). América Negra, 3: 171-189.

ARDILA, G.; POLITIS, G.

1992 La situación actual de los Nukak de la Amazonía Colombiana: Problemas y Perspectivas.
Revista de la Universidad Nacional de Colombia, 26: 2-6.

BAKER, M.

1997 Invisibility as a sympton of gender categories in archaeology. J. Moore; E. Scott (Eds.) Invisible People and Processes. Writing Gender and Childhood into European Archaeology. London, Leicester University Press: 183-191. 
POLITIS, G.G. La actividad infantil en la producción del registro arqueológico de cazadores-recolectores. Rev. do Museu de Arqueologia e Etnologia, São Paulo, Suplemento 3: 263-283, 1999.

BINFORD, L.

1977 Forty-seven Trips: A Case Study in the Character of Archaeological Formation Process. R.V.S. Wright (Ed.) Stone Tools as Cultural Markers. Canberra, Australian Institute of Aboriginal Studies: 24-36.

1978 Nunamiut Ethnoarchaeology. New York: Academic Press.

1979 Organization and Formation Processes: Looking at Curated Technologies. Journal of Anthropological Research, 35 (3): 255-273.

BODÚ, P.; KARLIN, C; PLOUX, S.

1990 Who is who? The Magdalenian flintkanppers of Pincevent. C. Cziesla; S. Eichoff; N. Arts; D. Winter (Eds.) The Big Puzzle. Bonn, Holos: 143-163.

BONNICHSEN, $\mathbf{R}$.

1973 Millie's Camp: an experiment in archaeology. World Archaeology, 4: 277-291.

BÓRMIDA, M.

1973 Ergon y mito. Una hermenéutica de la cultura material de los Ayoreo del Chaco Boreal. Scripta Ethnologica, 1 (1): 9-68.

BOURKE, J.

1891 Arrows and Arrow-makers. Remarks. American Anthropology 4: 71-74.

CABRERA, G; FRANKY Y MAHECHA, C.

1994 Aportes a la Etnografía de los Nukak y su lengua - Aspectos sobre Fonología Segmental. Trabajo de Grado para optar al título de Antropólogo. Universidad Nacional, Santafé de Bogotá.

CÁRDENAS, D.; POLITIS, G.

Territorio, movilidad, etnobotánica y manejo del bosque de los Nukak Orientales (Amazonía Colombiana). Boletín del Museo del Oro. Santafé de Bogotá.

CAYCEDO TURRIAGO, J.

1993 Los Nukak: transformaciones socioculturales y articulación étnica en una situación regional. F. Correa (Ed.) Encrucijadas de Colombia Amerindia. Santafé de Bogotá: Instituto Colombiano de Antropología: 141-159.

CHAMBERLAIN, A.

1997 Commentary: Missing stages of life - towards the perception of children in archaeology. J. Moore y E. Scott (Eds.) Invisible People and Processes. Writing Gender and Childhood into European Archaeology. London, Leicester University Press: 248-250.

CHAVES, M.; WIRPSA, L.

1988 Aparecen los Nukak. Noticias Antropológicas, 89. Sociedad Antropológica Colombiana, Santafé de Bogotá.

CLASTRES, $P$.

1998 Chronicle of the Guayaki Indians. New York: Faber and Faber.

CLASSEN, C. (Ed.)

1992 Exploring Gender Through Archaeology. Madison, Wisconsin: Prehistory Press.
CONKEY, M.W.; SPECTOR, J.

1984 Archaeology and the study of gender. M. Schiffer (Ed.) Advances in Archaeological Method and Theory, New York, 7: 1-38.

CONKEY, M.; GERO, J.

1991 Tension, Pluralities, and the Engendering Archaeology: An Introduction to Women and Prehistory. J. Gero; M.W. Conkey (Eds.) Engendering Archaeology. Women and Prehistory. Oxford, Blakwell: 3-30.

DRAPER, P.

1976 Social and economic constraints on child life among the !Kung. R. Lee; I. De Vore (Eds.) Kalahari hunter-gatherers. Cambridge, Harvard University Press: 199-217.

DRAPER, P.; CASHDAN, E.

1989 Technological change and child behaviour among the !Kung. Ethnology, 27 (4): 339-365.

FINLAY, N.

1997 Kid knapping: the missing children in lithic analysis. J. Moore; E. Scott (Eds.) Invisible People and Processes. Writing Gender and Childhood into European Archaeology. London, Leicester University Press: 203-212.

FISHER, A.

1990 On being a pupil flinknapper of 11.000 years ago. E. Cziesla; S. Eichoff; N. Arts; D. Winters (Eds.) The Big Puzzle. Bonn, Holos: 447-464.

FRANKY, C; CABRERA, G.; MAHECHA, D.

1995 Demografia y movilidad socio-espacial de los Nukak. Documento de Trabajo 2. Fundación Gaia Amazonas, Santafé de Bogotá, Colombia. GALLARDO, C.B.

1910 Tierra del Fuego. Los Onas. Buenos Aires.

GERO, J.

1991 Genderlithic: Women s Roles in Stone Tool Production. J. Gero; M. Conkey (Eds.) Engendering Archaeology. Women in Prehistory. Oxford: Blakwell.

GRADIN, C.; AGUERRE, A.

1983 Arte rupestre del "Area la Martita" Sección A del Departamento Magallanes. Provincia de Santa Cruz. Relaciones, 15 (N.S.): 195-223.

GRINNELL, G.

1923 The Cheyenne Indians: Their History and Ways of Life, 2 vols. New Haven: Yale University Press.

GUALTEROS, I.

Estudio breve sobre la cultura material de los $\mathrm{Nu}-$ kak. Asociación Nuevas Tribus de Colombia. 15 pp. Ms.

GUSINDE, $M$.

1982 Los Indios de Tierra del Fuego. Los Selknam. Centro Argentino de Etnologia Americana.

1987 Los Indios de Tierra del Fuego. Los Yámanas. Centro Argentino de Etnología Americana.

HAMMOND, G.; HAMMOND, N.

1981 Child's Play: A Distorting Factor in Archaeological Distribution. American Antiquity, 64: 634-636. 
POLITIS, G.G. La actividad infantil en la producción del registro arqueológico de cazadores-recolectores. Rev. do Museu de Arqueologia e Etnologia, São Paulo, Suplemento 3: 263-283, 1999.

HARKE, H.

1989 Knives in early Saxon burials length and age at death. Medieval Archaeology, 33: 144-148.

\section{HOLMBERG, A.}

1978 Nómadas del arco largo. Los Sirionó del Oriente boliviano. Instituto Indigenista Interamericano. Ediciones Especiales 77.

JAMES A.; PROUT, A.

1990 Constructing and Reconstructing Childhood. Basingstocke: The Farmel Press.

JONES, $\mathrm{S}$.

1997 The Archaeology of Ethnicity. Constructing identities in the past and present. New York: Routledge.

KOCH-GRÜNBERG, T.

1906 Die Makú. Anthropos, 1: 877-906.

LESIK, $\mathrm{K}$.

1997 Re-engendering gender: some theoretical and methodological conserns on burgeoning archaoelogical pursuit. J. Moore; E. Scott (Eds.) Invisible People and Processes. Writing Gender and Childhood into European Archaeology. London, Leicester University Press: 31-41.

LILLEHAMMER, G.

1989 A Child is Born. The Child's World in an Archaeological Perspective. Norwegian Archaeological Review, 22 (2): 89-105.

MENGONI GOÑALONS, G.

Mesa Redonda: Actores en escena: comportamiento social y el registro arqueológico. Soplando en el viento: Actas de las Terceras Jornadas de Arqueología de la Patagonia. Universidad del Comahue e INALP. (e.p.)

METRAUX, A

1948 The hunting and gathering people of the Rio Negro Basin. J. Steward (Ed.) Handbook of Southamerican Indians, vol. 3. Washington, Smithsonian: 861-867.

MONDRAGÓN, H.

Estudio para el establecimiento de un programa de defensa de la comunidad indígena $\mathrm{Nu}$ kak. Informe final presentado al programa de Rehabilitación Nacional (PNR) de la Presidencia de la República de Colombia. 48 pp. Ms.

MOORE, J.; SCOTT, E. (Eds.)

1997 Invisible People and Processes. Writing Gender and Childhood into European Archaeology. London: Leicester University Press.

MUSTERS, G.

1997 Vida entre los Patagones. Buenos Aires: Elefante Blanco.

PARK, R.

1998 Size counts: the miniature archaelogy of childhood in Inuit societies. Antiquity, 72 (276): 269-281.

POLITIS, G.

1992 La Arquitectura del Nomadismo en la Amazonía Colombiana. Proa, 412: 11-20.

1996a Moving to produce. Nukak mobility and settlements patterns in Amazonia. World Archaeology, 27 (3): 492-510. 1996b Nukak. Santafé de Bogotá: Instituto Amazónico de Investigaciones Científicas SINCHI.

1996c La formación de sitios de cazadores-recolectores en las Tierras Bajas Sudamericanas: un caso de estudio etnoarqueológico. Ponencia presentada en el Simposio Internacional Arqueología de Tierras Bajas. Montevideo, Uruguay, abril de 1996.

1998 Arqueología de la infancia. Una perspectiva etnoarqueológica. Trabajos de Prehistoria, Madrid, España, 55 (2): 5-19.

POLITIS G.; MARTÍNEZ, G.

1992 La Subsistencia Invernal de un Grupo de los Nukak Nor-Occidental del Guaviare. Trabajo presentado en el 6to. Congreso Nacional de Antropología de Colombia. Simposio Pasado y Presente de los Cazadores Recolectores en América del Sur. Santafé de Bogotá, Colombia, julio de 1992.

POLITIS, G.; RODRÍGUEZ J., J.

1994 Algunos aspectos de la subsistencia de los Nukak de la Amazonía Colombiana. Colombia Amazónica, 7 (1-2): 169-207.

POLITIS, G.; SAUNDERS, N.

Archaeological correlates of ideological activity: Food taboos and spirit animal in an Amazonian hunter-gatherer society. P. Miracle (Ed.) "Consuming Passions. Consuming Things" Cambridge, Inglaterra: Mc Donald Institute. (e.p.)

POLITIS, G.; MARTÍNEZ, G.; RODRÍGUEZ, J.

1997 Caza, recolección y pesca como estrategia de explotación de recursos en forestas tropicales lluviosas: los Nukak de la Amazonía colombiana. Revista Española de Antropología Americana, 27: 167-197.

REICHEL-DOLMATOFF, G.

1967 A brief report on urgent ethnological research in the Vaupes area. Colombia, South America. Bulletin of International Committee on urgent anthropological research, 9: 53-62.

REID, $\mathrm{H}$.

1979 Some aspects of movement, growth, and change among the Hupdu Makú indians of Brazil. Ph.D. Dissertation. Cambridge University, Cambridge.

REINA, L

Informe de comisión entre la comunidad indígena Nukak, Corregimiento de Calamar, Guaviare. Instituto Colombiano de Antropología. Santafé de Bogotá. Ms.

1990 Actividades relacionadas con los Nukak. Mopa-Mopa, Instituto Andino de Artes Populares. Pasto, 5: 17-25

RIVAS, P.; OCAMPO. C.

1997 Dinámica sociocultural de las poblaciones asentadas en el ecosistema Litoral Santa Augusta de Quintay, Proyecciones regionales e interregionales y el Período Medio de Chile Central. Trabajo presentado en el XIV Congreso Nacional de Arqueología Chilena, 13 al 18 de Octubre de 1997. Copiapó. 
POLITIS, G.G. La actividad infantil en la producción del registro arqueológico de cazadores-recolectores. Rev. do Museu de Arqueologia e Etnologia, São Paulo, Suplemento 3: 263-283, 1999.

\section{SHENNAN, S.}

1991 Tradition, Rationality, and Cultural Transmission. R. Preucel (Ed.) Processual and Postprocessual Archaeologies: Multiples Ways of Knowing the Past. Illinois, University of Carbondale: 197-208.

SOFAER-DEREVENSKI, J.

1994 Where are the Children? Accesing Children in the Past. Archaeological Review from Cambridge, 13 (2): 7-20.

1997 Age and gender at the site of Tiszapolgár-Basatanya, Hungary. Antiquity, 71 (274): 875-879.

SCOTT, E.

1997 Introduction: On the incompletness of archaeological narratives. J. Moore; E. Scott (Eds.) Invisible People and Processes. Writing Gender and Childhood into European Archaeology. London, Leicester University Press: 1-12.

\section{SILVERWOOD-COPE, P.}

1972 A contribution to the ethnography of the Colombian Makú. Tésis Doctoral. Cambridge: Cambridge University Press.

TOTH, N.; CLARK, D.; LIGABUE, G.

1992 Los últimos fabricantes de hachas de piedra. Investigación y Ciencia. Septiembre: 6-11.

\section{WIESSNER, P.}

1983 Style and ethnicity in the Kalahari San projectile point. American Antiquity, 50: 253-276.

\section{WIRPSA, L.; MONDRAGÓN, H.}

1988 Resettlement of Nukak Indians, Colombia. $\mathrm{Cul}$ tural Survival Quarterly, 12 (4): 36-40.

\section{WHITLEY, D. (Ed.)}

1998 Reader in Archaeological Theory. Post-Processual and Cognitive Approaches. London: Routledge. 